Generalized linear differential equations in a Banach space: continuous dependence on parameters and applications 



\title{
Generalized linear differential equations in a Banach space: continuous dependence on parameters and applications
}

\author{
Giselle Antunes Monteiro
}

Advisor: Profa. Dra. Márcia Cristina Anderson Braz Federson

Doctoral dissertation submitted to the Instituto de Ciências Matemáticas e de Computação - ICMC-USP, in partial fulfillment of the requirements for the degree of the Doctorate Program in Mathematics. REVISED COPY.

USP - São Carlos

February 2012 
Ficha catalográfica elaborada pela Biblioteca Prof. Achille Bassi e Seção Técnica de Informática, ICMC/USP, com os dados fornecidos pelo(a) autor(a)

\begin{tabular}{|c|c|}
\hline \multirow[t]{3}{*}{ M772g } & $\begin{array}{l}\text { Monteiro, Giselle Antunes } \\
\quad \text { Generalized linear differential equations in a } \\
\text { Banach space: continuous dependence on parameters } \\
\text { and applications / Giselle Antunes Monteiro; } \\
\text { orientadora Márcia Cristina Anderson Braz Federson. - } \\
\text { - São Carlos, 2012. } \\
\quad 81 \text { p. }\end{array}$ \\
\hline & $\begin{array}{l}\text { Tese (Doutorado - Programa de Pós-Graduação em } \\
\text { Matemática) -- Instituto de Ciências Matemáticas e } \\
\text { de Computação, Universidade de São Paulo, } 2012 \text {. }\end{array}$ \\
\hline & $\begin{array}{l}\text { 1. Generalized Differential Equations. } 2 \text {. } \\
\text { Kurzweil-Stieltjes integral. 3. Dynamic Equations on } \\
\text { Time Scales. 4. Functional Differential Equations. } \\
\text { I. Federson, Márcia Cristina Anderson Braz, orient. } \\
\text { II. Título. }\end{array}$ \\
\hline
\end{tabular}


"Oftentimes, it is madness more than genius that moves the world forward." (James Rollins) 



\section{Agradecimentos}

Gradeço, primeiramente, a minha orientadora Profa. Márcia Federson por seu otimismo
(com a matemática e com a vida) e por acreditar no meu potencial sempre, desde o primeiro momento.

Agradeço a banca examinadora pelas sugestões apresentadas que apontam tantas direções para futuras pesquisas.

Agradeço ao Prof. Ivo Machado da Costa do DM-UFSCar, meu conselheiro acadêmico e amigo desde a graduação. Agradeço à Profa. Roseli Fernandez do IME-USP, minha orientadora durante o Mestrado, por ter aberto tantas portas para mim (inclusive por me apresentar à Profa. Márcia).

Agradeço a minha família, minhas ‘mães’ Dora, Delmina, Carolina e Gonçalina, minhas ‘irmãs’ Vivian e Ivana, pelo amor e paciência, por compreenderem minhas ausências. Agradeço ainda os muitos mimos a cada retorno meu.

Agradeço a minha família em São Carlos, Maria Clara de Freitas e Thaize Reis; mais que amigas, verdadeiras irmãs. Obrigada por me lembrarem que não sou feita apenas de matemática.

Agradeço a minha grande amiga Andra Sara (in memoriam), que sempre me inspirou a dar o melhor de mim. Agradeço ao meu amigo Maico Guarnieri, por sempre me incentivar a escrever (matemática, entre outras coisas). Agradeço à Lilian Chiba, sempre me salvando da solidão nas conversas virtuais e nos livros emprestados. Agradeço à Maria Simone Kugeratski, para mim um 
exemplo de disciplina. Agradeço ao jovem Leandro Pimenta, pela companhia em tantos momentos. Agradeço ao meu amigo Fernando Cotinguiba, que sempre me avisa dos concursos, pela amizade aqui, em Águas de Lindóia, em Berlin, Dresden, Praga e, especialmente, em Munique.

Agradeço ao meu meniino, Marcos Vasconcelos, por ser sempre tão sublime comigo e pelo presente que me deste, um novo coração.

Agradeço às meninas da CPG, Ana Paula S. Fregona, Ana Carolina V. Murata, Laura Aparecida D. R. Turi e Lhaís Visentin, pela atenção dedicada (sempre dando um ‘jeitinho’ para as coisas funcionarem). Agradeço aos amigos e colegas do ICMC que partilharam comigo esse período, em especial, Alisson Rafael, Eduard Toon, Jaqueline Godoy, Patricia Hilário e Paulo Carvalho. Agradeço à Suzete Maria, minha irmãzinha acadêmica, pelo especial apoio em tantos momentos decisivos. Agradeço também ao companheiro de estudos em teorias de integração Pedro Kaufmann pela sinceridade sempre.

Por fim, agradeço o apoio financeiro do CNPq e CAPES (PDEE) nas estapas iniciais deste trabalho. Agradeço ainda a FAPESP pelo suporte financeiro durante a etapa final de execução deste trabalho.

I am thankful for the time I spent with Professor Štefan Schwabik (in memoriam), while he was here in Brazil in 2008, for the fruitful conversation about Math. I am thankful and honoured to be given the chance to meet Professor Jaroslav Kurzweil, an inspiring Mathematician.

I am gratiful to my dears Anna Maria Adamska and Stancho Stanchev, for the companionship during my stay in Prague.

Finally, my special thanks to Professor Milan Tvrdý, who accepted me as a visiting graduate student at the Academy of Sciences of the Czech Republic. For all care devoted to me (including our lunchtimes) and all academic things I have learnt; for all these: děkuji! 
The purpose of this work is to investigate continuous dependence on parameters for generalized linear differential equations in a Banach spacevalued setting. More precisely, we establish a theorem inspired by the classical continuous dependence result due to Z . Opial. In addition, our second outcome extends, to Banach spaces, the result proved by M. Ashordia in the framework of finite dimensional generalized linear differential equations. Roughly speaking, the continuous dependence derives from assumptions of uniform convergence of the functions in the right-hand side of the equations, together with the uniform boundedness of variation of the linear terms. Furthermore, applications of these results to dynamic equations on time scales and also to functional differential equations are proposed.

Besides these results on continuous dependence, we complete the theory of abstract Kurzweil-Stieltjes integration so that it is well applicable for our purposes in generalized linear differential equations. In view of this, our contributions are related not only to differential equations but also to the abstract Kurzweil-Stieltjes integration theory itself.

The new results presented in this work are contained in the papers [26] and [27], both accepted for publication. 

O objetivo deste trabalho é investigar a dependência contínua de soluções em relação a parâmetros para equações diferenciais lineares generalizadas no contexto de espaços de Banach. Mais precisamente, apresentamos um teorema inspirado no resultado clássico de dependência contínua obtido por Z. Opial. Nosso segundo resultado estende, para espaços de Banach, o provado por M. Ashordia no contexto de equações diferenciais lineares generalizadas em dimensão finita. Em linhas gerais, a dependência contínua decorre da convergência uniforme das funções à direita das equações, juntamente com a limitação uniforme da variação dos termos lineares. No mais, são propostas aplicações desses resultados em equações dinâmicas em escalas temporais e também em equações diferenciais funcionais.

Além dos resultados em dependência contínua, completamos à teoria de integração abstrata de Kurzweil-Stieltjes de modo que esta se adeque aos nossos propósitos em equações diferenciais lineares generalizadas. Assim, nossas contribuições dizem respeito não apenas a equações diferenciais, mas também a teoria de integração abstrata de Kurzweil-Stieltjes em si.

Os resultados originais apresentados neste trabalho estão contidos nos artigos [26] e [27], ambos aceitos para publicação. 

1 Kurzweil-Stieltjes integration theory in Banach space 15

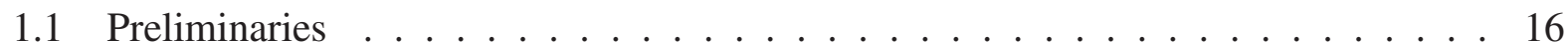

1.2 Kurzweil-Stieltjes integral . . . . . . . . . . . . . . . . 20

2 Generalized linear differential equations in Banach space $\quad 35$

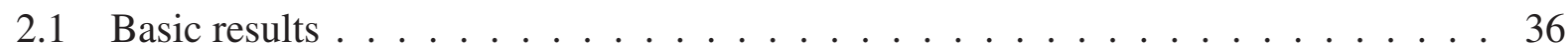

2.2 Continuous dependence on parameters: variations bounded by a weight . . . . . . 39

2.3 Continuous dependence on parameters: uniformly bounded variation . . . . . . . . 43

3 Applications to dynamic equations on time scales $\quad 51$

3.1 Basic results and definitions $\ldots \ldots \ldots \ldots \ldots \ldots$

3.2 Continuous dependence for dynamical equations on time scales . . . . . . . . . . 54

4 Applications to functional differential equations 
4.1 RFDEs and generalized differential equations . . . . . . . . . . . . . 59

4.2 Continuous dependence for linear functional differential equations . . . . . . . . . 67 


\section{Introduction}

The theory of generalized ordinary differential equations (generalized ODEs) has its roots in the work of the Czech mathematician Jaroslav Kurzweil, see [24] and [25]. In particular, it was a problem on continuous dependence on parameters which inspired J. Kurzweil to define such notion of equations in 1957.

After Jaroslav Kurzweil, the problem of continuous dependence on parameters for generalized ODEs has been investigated by several authors as, for instance, Š. Schwabik [32], M. Ashordia [3], D. Fraňková [12], M. Tvrdý [43], Z. Halas and M. Tvrdý [18]. Although, it seems that, up to now, only in [1] the problem was treated for infinite dimensional spaces.

The theory of generalized ODEs in a general Banach space setting enables the investigation of continuous and discrete systems from a common understanding. Indeed, the existence of certain correspondence between generalized ODEs and other types of differential systems, such as, functional differential equations, equations with impulses, dynamic equations on time scales, are well-known facts. See, for instance, [10], [23], [29], [32] or [39]. All these together represent good reasons for revisiting the problem of continuous dependence on parameters for generalized ODEs, once the existing correspondences may allow us to translate the obtained results for other theories of differential equations. 
In the present work, we deal with integral equations of the form

$$
x(t)=\widetilde{x}+\int_{a}^{t} \mathrm{~d}[A(s)] x(s)+f(t)-f(a), \quad t \in[a, b],
$$

where $-\infty<a<b<\infty, X$ is a Banach space, $\widetilde{x} \in X, A:[a, b] \rightarrow X$ is a function of bounded variation on $[a, b], f:[a, b] \rightarrow X$ is regulated on $[a, b]$ and the integral is understood in the KurzweilStieltjes' sense.

The equation (I) is a special case of generalized ODEs, namely a linear equation, in its integral form. Concerning this type of equations for functions with values in a Banach space, the contributions by Š. Schwabik in [33] and [34] are essential.

Our aim in this work is to present new results on continuous dependence on parameters which are valid in a very general Banach space setting, for generalized ODEs of the type (I).

In order to do that, a better knowledge of abstract Kurzweil-Stieltjes-type of integral is needed. This is the content of Chapter 1, where, together with the basic theory of such integral, we present also some new results which will be important throughout the development of the thesis. We mention that the new results contained in this chapter are described in the paper [26], to appear.

In the second chapter, we study equation (I). Some well-known properties of solutions of generalized linear differential equation are given in the first section. In the remaining of the chapter, we establish two new results on continuous dependence on parameters. In summary, such results generalizes in some aspects the work by M. Ashordia, Z. Opial, Š. Schwabik, Z. Halas and M. Tvrdý, in their respective papers [3], [30], [32] and [18]. Indeed, our Theorem 2.4, treating homogeneous equations, was inspired in the result given Z. Opial for linear ordinary differential equation (cf. [30, Theorem 1]). In addition, our main theorem in Section 2.3 has general assumptions in comparison with the continuous dependence result found in [32]. This fact is pointed out in Propositions 2.11 and 2.12. The new results described in this chapter are contained in the paper [27], to appear.

The following two chapters are devoted to applications of the results obtained in Chapter 2 to other differential equations theories. In Chapter 3, we obtain new results on continuous dependence on parameters for linear dynamic equations on time scales via generalized ODEs. Concerning the results presented in this chapter, let us mention that, Theorems 14 and 16 from [39], when restricted to the linear case, follow as a consequence of our Theorem 3.3. 
The Chapter 4 concerns functional differential equations. At first, we show that, under an assumption of Lipschitz-type, the existence of solution of the linear problem in functional differential equation is guaranteed by existence of solution of the corresponding generalized linear differential equation. Such procedure is a particular case of the one described by M. Federson and Š. Schwabik in [10]. We conclude the chapter using the established correspondence to derive new continuous dependence results for linear functional differential equations. It is worth highlighting that our Theorem 4.4, provide a more general result than previous one, such as [10, Theorem 4.1] and [11, Theorem 3.4] when restricted to linear equations with no impulses. 



$\left(\frac{10}{1}\right.$

\section{Kurzweil-Stieltjes integration theory in Banach space}

The extension to Banach space-valued functions of integrals which are based on Riemann type sums, such as the Kurzweil-Henstock and the McShane integrals, have been studied by many authors. Among other contributions it is worth highlighting the monograph by Š. Schwabik and G. Ye [37] which studies these type of integrals and their connections with the classic integrals due to Bochner and Pettis. Concerning integrals of Stieltjes-type, C. S. Hönig presented a quite complete study in [22] dealing with the interior integral, also known as Dushnik integral.

In this work, we deal with integral equations in the framework of Kurzweil-Stieltjes integral for Banach space valued-functions, also called abstract Perron-Stieltjes integral. This chapter is devoted to the study of these integral. We collect some known results (see [33]) and we complete the theory in such a way that the results we obtain are used in the following chapters. In particular, we establish an Integration by Parts result under assumptions covered neither by Š. Schwabik [36] nor 
by K. M. Naralenkov [28]. Enclosing this chapter, two Substitution theorems, which complement those treated by M. Federson in [7], are presented.

\subsection{Preliminaries}

In this section, we introduce the basic notions related to the abstract Kurzweil-Stieltjes integral. The main references for this section are [22] and [33].

Let $\mathbb{N}=\{1,2, \ldots\}$ and $\mathbb{R}$ be the set of real numbers.

Throughout this work, $X$ is a Banach space and $L(X)$ is the Banach space of bounded linear operators on $X$. By $\|\cdot\|_{X}$ and $\|\cdot\|_{L(X)}$ we denote the norm in $X$ and the usual operator norm in $L(X)$ respectively. In particular, if $X=\mathbb{R}^{m}$, for some fixed $m \in \mathbb{N}$, the norm will be denoted by single bars, $|\cdot|$.

Assuming $-\infty<a<b<+\infty,[a, b]$ stands for the closed interval, $(a, b)$ is its interior and $(a, b],[a, b)$ are the corresponding half-closed intervals.

A division of $[a, b]$ is any finite set $D=\left\{\alpha_{0}, \alpha_{1}, \ldots, \alpha_{\nu(D)}\right\}$ with

$$
a=\alpha_{0}<\alpha_{1}<\ldots<\alpha_{\nu(D)}=b .
$$

The set of all divisions of $[a, b]$ is denoted by $\mathcal{D}[a, b]$.

We say $f:[a, b] \rightarrow X$ is a finite step function on $[a, b]$, if there is a division $D=\left\{\alpha_{0}, \ldots, \alpha_{\nu(D)}\right\}$ of $[a, b]$ such that $f$ is constant on $\left(\alpha_{j-1}, \alpha_{j}\right)$, for each $j=1,2, \ldots, \nu(D)$.

For an arbitrary function $f:[a, b] \rightarrow X$,

$$
\operatorname{var}_{a}^{b} f=\sup \left\{\sum_{j=1}^{\nu(D)}\left\|f\left(\alpha_{j}\right)-f\left(\alpha_{j-1}\right)\right\|_{X} ; D \in \mathcal{D}[a, b]\right\}
$$

denotes the variation of $f$ on $[a, b]$. When $\operatorname{var}_{a}^{b} f<\infty$, the function $f$ is of bounded variation on $[a, b]$. By $B V([a, b], X)$ we denote the Banach space of all $X$-valued functions of bounded variation on $[a, b]$, equipped with the norm $\|f\|_{B V}=\|f(a)\|_{X}+\operatorname{var}_{a}^{b} f$. 
Assume $\mathcal{B}=(L(X), X, X)$ is the bilinear triple obtained using the bilinear form

$$
B: L(X) \times X \rightarrow X, \quad B(A, x)=A x \in X, \quad \text { for } A \in L(X) \text { and } x \in X
$$

With respect to this bilinear triple $\mathcal{B}$, we will now present general notions of bounded variation and regulated functions.

Given $F:[a, b] \rightarrow L(X), f:[a, b] \rightarrow X$ and a division $D=\left\{\alpha_{0}, \alpha_{1}, \ldots, \alpha_{\nu(D)}\right\}$ of the interval $[a, b]$, consider

$$
V_{a}^{b}(F, D)=\sup \left\{\left\|\sum_{j=1}^{\nu(D)}\left[F\left(\alpha_{j}\right)-F\left(\alpha_{j-1}\right)\right] y_{j}\right\|_{X} ; y_{j} \in X,\left\|y_{j}\right\|_{X} \leq 1\right\}
$$

and

$$
v_{a}^{b}(f, D)=\sup \left\{\left\|\sum_{j=1}^{\nu(D)} F_{j}\left[f\left(\alpha_{j}\right)-f\left(\alpha_{j-1}\right)\right]\right\|_{X} ; F_{j} \in L(X),\left\|F_{j}\right\|_{L(X)} \leq 1\right\} .
$$

We define the semi-variation of $F$ on $[a, b]$ as

$$
(\mathcal{B}) \operatorname{var}_{a}^{b} F=\sup \left\{V_{a}^{b}(F, D) ; D \in \mathcal{D}[a, b]\right\}
$$

(also known as $\mathcal{B}$-variation of $F$, cf. [33]Analogously, we define the $\mathcal{B}$-variation of $f$ on $[a, b]$ as

$$
(\mathcal{B}) \operatorname{var}_{a}^{b} f=\sup \left\{v_{a}^{b}(f, D) ; D \in \mathcal{D}[a, b]\right\}
$$

The notation $(\mathcal{B}) B V([a, b], L(X))$ stands for the set of all functions $F:[a, b] \rightarrow L(X)$ of bounded $\mathcal{B}$-variation on $[a, b]$, that is, $(\mathcal{B}) \operatorname{var}_{a}^{b}(F)<\infty$, in which we consider the norm

$$
\|F\|_{S V}=\|F(a)\|_{L(X)}+(\mathcal{B}) \operatorname{var}_{a}^{b} F .
$$

It is worth highlighting that, in the finite dimensional case, the concepts of bounded variation and bounded $\mathcal{B}$-variation are equivalent.

Now, let us recall that a function $f:[a, b] \rightarrow X$ is regulated on $[a, b]$, if the one-sided limits

$$
f(t-)=\lim _{s \rightarrow t-} f(s) \quad \text { and } \quad f(t+)=\lim _{s \rightarrow t+} f(s)
$$

exist at every point of $t \in[a, b]$ (by convention $f(a-)=f(a)$ and $f(b+)=f(b)$ ). For $t \in[a, b]$, we put $\Delta^{+} f(t)=f(t+)-f(t)$ and $\Delta^{-} f(t)=f(t)-f(t-)$. 
By $G([a, b], X)$ we denote the set of all regulated functions $f:[a, b] \rightarrow X$, which is a Banach space when endowed with the usual supremum norm

$$
\|f\|_{\infty}=\sup \left\{\|f(t)\|_{X} ; t \in[a, b]\right\} .
$$

Clearly, $\mathcal{C}([a, b], X) \subset G([a, b], X)$, where $\mathcal{C}([a, b], X)$ is the Banach space of all continuous functions from $[a, b]$ to $X$.

A function $F:[a, b] \rightarrow L(X)$ is $\mathcal{B}$-regulated on $[a, b]$ (or weakly regulated on $[a, b]$, cf. [22]), if for every $x \in X$, with $\|x\|_{X} \leq 1$, the function $t \in[a, b] \longmapsto F(t) x$ is regulated. Similarly, $f:[a, b] \rightarrow X$ is $\mathcal{B}$-regulated on $[a, b]$, if the function $t \in[a, b] \longmapsto T f(t)$ is regulated for all $T \in L(X)$ with $\|T\|_{L(X)} \leq 1$.

The set of all $\mathcal{B}$-regulated operator valued functions is denoted by $(\mathcal{B}) G([a, b], L(X))$.

The next proposition presents some interesting properties of the classes of functions described before.

Proposition 1.1. The following assertions hold:

(i) [22, Theorem I.3.1] Every regulated function $f:[a, b] \rightarrow X$ is the uniform limit of finite step functions.

(ii) [22, Corollary I.3.2.b] The set of all discontinuities of a regulated function $f:[a, b] \rightarrow X$ is at most countable.

(iv) [21, Theorem I.2.7] $B V([a, b], L(X)) \subset G([a, b], L(X))$.

(v) [33, Proposition 1] If $F \in B V([a, b], L(X))$, then $F \in(\mathcal{B}) B V([a, b], L(X))$ and

$$
(\mathcal{B}) \operatorname{var}_{a}^{b} F \leq \operatorname{var}_{a}^{b} F
$$

(vi) [33, Proposition 3] $G([a, b], L(X)) \subset(\mathcal{B}) G([a, b], L(X))$.

The next lemma presents an estimate which is well-known in the finite dimensional case for Banach space-valued functions. 
Lemma 1.2. If $g \in B V([a, b], X)$, then $\sum_{t \in[a, b)}\left\|\Delta^{+} g(t)\right\|_{X}+\sum_{t \in(a, b]}\left\|\Delta^{-} g(t)\right\|_{X} \leq \operatorname{var}_{a}^{b} g$.

Proof. By Proposition 1.1 (ii), the set of discontinuties of $g$ in the interval $(a, b)$ can be written as $\left\{t_{k} \in X ; k \in \mathbb{N}\right\}$. We can assume, without loss of generality, that $t_{k}<t_{k+1}, k \in \mathbb{N}$. For each $n \in \mathbb{N}$, define

$$
S_{n}=\left\|\Delta^{+} g(a)\right\|_{X}+\left\|\Delta^{-} g(b)\right\|_{X}+\sum_{k=1}^{n}\left[\left\|\Delta^{-} g\left(t_{k}\right)\right\|_{X}+\left\|\Delta^{+} g\left(t_{k}\right)\right\|_{X}\right] .
$$

Let $\varepsilon>0$ and $n \in \mathbb{N}$ be given. Since $g \in G([a, b], X)$ (see Proposition 1.1 (iv)), for each $k=1,2, \ldots, n$, choose $\delta_{k}>0$ in such a way that

$$
\left\|g\left(t_{k}+\delta_{k}\right)-g\left(t_{k}+\right)\right\|_{X}<\frac{\varepsilon}{4(n+1)}, \quad\left\|g\left(t_{k}-\delta_{k}\right)-g\left(t_{k}-\right)\right\|_{X}<\frac{\varepsilon}{4(n+1)}
$$

and $\left[t_{k}-\delta_{k}, t_{k}+\delta_{k}\right] \cap\left\{t_{1}, t_{2}, \ldots, t_{n}\right\}=\left\{t_{k}\right\}$. Analogously, let $\delta_{0}>0$ be such that

$$
\left\|g\left(a+\delta_{0}\right)-g(a+)\right\|_{X}<\frac{\varepsilon}{4}, \quad\left\|g(b-)-g\left(b-\delta_{0}\right)\right\|_{X}<\frac{\varepsilon}{4} .
$$

and also $a+\delta_{0}<t_{1}$ and $b-\delta_{0}>t_{n}$. It follows that

$$
\begin{aligned}
S_{n} \leq & \left(\left\|g(a+)-g\left(a+\delta_{0}\right)\right\|_{X}+\left\|g\left(a+\delta_{0}\right)-g(a)\right\|_{X}\right) \\
& +\sum_{k=1}^{n}\left\|g\left(t_{k}+\right)-g\left(t_{k}+\delta_{k}\right)\right\|_{X}+\sum_{k=1}^{n}\left\|g\left(t_{k}+\delta_{k}\right)-g\left(t_{k}\right)\right\|_{X} \\
& +\sum_{k=1}^{n}\left\|g\left(t_{k}-\right)-g\left(t_{k}-\delta_{k}\right)\right\|_{X}+\sum_{k=1}^{n}\left\|g\left(t_{k}-\delta_{k}\right)-g\left(t_{k}\right)\right\|_{X} \\
& +\left(\left\|g(b)-g\left(b-\delta_{0}\right)\right\|_{X}+\left\|g\left(b-\delta_{0}\right)-g(b-)\right\|_{X}\right) \\
< & \frac{\varepsilon}{4}+\left\|g\left(a+\delta_{0}\right)-g(a)\right\|_{X}+\frac{n \varepsilon}{4(n+1)}+\sum_{k=1}^{n}\left\|g\left(t_{k}+\delta_{k}\right)-g\left(t_{k}\right)\right\|_{X} \\
& +\frac{n \varepsilon}{4(n+1)}+\sum_{k=1}^{n}\left\|g\left(t_{k}\right)-g\left(t_{k}-\delta_{k}\right)\right\|_{X}+\left\|g(b)-g\left(b-\delta_{0}\right)\right\|_{X}+\frac{\varepsilon}{4}
\end{aligned}
$$

that is, for each $n \in \mathbb{N}$,

$$
\begin{aligned}
S_{n}< & \varepsilon+\left(\left\|g\left(a+\delta_{0}\right)-g(a)\right\|_{X}+\sum_{k=1}^{n}\left\|g\left(t_{k}+\delta_{k}\right)-g\left(t_{k}\right)\right\|_{X}\right) \\
& +\left(\sum_{k=1}^{n}\left\|g\left(t_{k}\right)-g\left(t_{k}-\delta_{k}\right)\right\|_{X}+\left\|g(b)-g\left(b-\delta_{0}\right)\right\|_{X}\right) .
\end{aligned}
$$


Hence $S_{n} \leq \operatorname{var}_{a}^{b} g$, for all $n \in \mathbb{N}$. Since

$$
\sum_{t \in[a, b)}\left\|\Delta^{+} g(t)\right\|_{X}+\sum_{t \in(a, b]}\left\|\Delta^{-} g(t)\right\|_{X}=\lim _{n \rightarrow \infty} S_{n}
$$

the expected estimate holds.

\subsection{Kurzweil-Stieltjes integral}

Concerning Kurzweil-Stieltjes integral for functions taking values in a Banach space, the contribuitions of M. Federson in [7] and also Š. Schwabik in [33] and [36] are essential. In this section, we collect and extend some of these results.

Let us recall the definition of the Kurzweil-Stieltjes integral.

As usual, a partition of a interval $[a, b]$ is a tagged division, $P=(\xi, D)$, where $D \in \mathcal{D}[a, b]$, with $D=\left\{\alpha_{0}, \alpha_{1}, \ldots, \alpha_{\nu(D)}\right\}$ and $\xi_{j} \in\left[\alpha_{j-1}, \alpha_{j}\right], j=1,2, \ldots, \nu(D)$. Alternatively, we can write $P=\left(\xi_{j},\left[\alpha_{j-1}, \alpha_{j}\right]\right)$ instead of $P=(\xi, D)$.

A gauge on $[a, b]$ is any positive function $\delta:[a, b] \rightarrow(0, \infty)$. Further, given a gauge $\delta$ on $[a, b]$, a partition $P=\left(\xi_{j},\left[\alpha_{j-1}, \alpha_{j}\right]\right)$ is called $\delta$-fine, if

$$
\left[\alpha_{j-1}, \alpha_{j}\right] \subset\left(\xi_{j}-\delta\left(\xi_{j}\right), \xi_{j}+\delta\left(\xi_{j}\right)\right), \quad \text { for } j=1,2, \ldots, \nu(P) \text {. }
$$

Given an arbitrary gauge $\delta$ on $[a, b]$, there exists at least one $\delta$-fine partition of $[a, b]$. This result is known as Cousin's Lemma, see [19, Theorem 4.1], for instance.

Definition 1.3. Let $F:[a, b] \rightarrow L(X)$ and $g:[a, b] \rightarrow X$ be given .

(i) The Kurzweil-Stieltjes integral (or shortly $K S$-integral) of $F$ with respect to $g$ on $[a, b]$ exists, if there is $I \in X$ satisfying: for every $\varepsilon>0$, there exists a gauge $\delta$ on $[a, b]$ such that

$$
\|S(F, \mathrm{~d} g, P)-I\|_{X}<\varepsilon \text { for all } \delta \text {-fine partitions } P \text { of }[a, b]
$$

where $S(F, \mathrm{~d} g, P)=\sum_{j=1}^{\nu(P)} F\left(\xi_{j}\right)\left[g\left(\alpha_{j}\right)-g\left(\alpha_{j-1}\right)\right]$, for $P=\left(\xi_{j},\left[\alpha_{j-1}, \alpha_{j}\right]\right)$. In this case, we write $I=\int_{a}^{b} F \mathrm{~d}[g]$. 
(ii) Similarly, $J \in X$ is the $K S$-integral of $g$ with respect to $F$ on $[a, b]$, we write $J=\int_{a}^{b} \mathrm{~d}[F] g$, if for every $\varepsilon>0$, there exists a gauge $\delta$ on $[a, b]$ such that

$$
\|S(\mathrm{~d} F, g, P)-J\|_{X}<\varepsilon \quad \text { for all } \delta \text {-fine partitions } P \text { of }[a, b]
$$

where $S(\mathrm{~d} F, g, P)=\sum_{j=1}^{\nu(P)}\left[F\left(\alpha_{j}\right)-F\left(\alpha_{j-1}\right)\right] g\left(\xi_{j}\right)$, for $P=\left(\xi_{j},\left[\alpha_{j-1}, \alpha_{j}\right]\right)$.

Let us mention that, when restricted to Euclidian spaces, the integral defined above corresponds to a particular case of the generalized Perron integral extensively studied in [32].

A typical situation arises when $X$ is the set real numbers and $F, g:[a, b] \rightarrow \mathbb{R}$. Taking $g(t)=t$, for all $t \in[a, b]$, we have

$$
S(F, \mathrm{~d} g, P)=\sum_{j=1}^{\nu(P)} F\left(\xi_{j}\right)\left(\alpha_{j}-\alpha_{j-1}\right), \quad \text { for all partitions } P \text { of }[a, b],
$$

which corresponds to the usual Riemann sum. In this case, the integral from Definition 1.3 (i) is actually the Kurzweil-Henstock integral (see [14, Definition 9.3]).

Another important relation to be pointed out concerns the Bochner-Stieltjes integral for Banach space-valued functions. It is known that the set of Bochner integrable functions is strictly contained in the set of Henstock integrable ones (cf. [8]). Clearly, then, every Bochner-Stieltjes integrable function is $K S$-integrable (see [31]).

Furthermore, the $K S$-integral extends the Riemann-Stieltjes integral defined in the Banach space setting (see [21]). More precisely:

If the Riemann-Stieltjes integral (RS) $\int_{a}^{b} F \mathrm{~d}[g]$ exists, then the KS-integral $\int_{a}^{b} F \mathrm{~d}[g]$ also exists and both integrals coincide.

Regarding the $K S$-integral given by Definition 1.3, the properties of linearity and additivity with respect to adjacent intervals hold in this abstract context (cf. [33]). Some further results are summarized in the next proposition.

Proposition 1.4. Let $F:[a, b] \rightarrow L(X)$ and $g:[a, b] \rightarrow X$. 
(i) [33, Proposition 10] If $F \in(\mathcal{B}) B V([a, b], L(X))$ and g are such that the integral $\int_{a}^{b} \mathrm{~d}[F] g$ exists, then

$$
\left\|\int_{a}^{b} \mathrm{~d}[F] g\right\|_{X} \leq\left((\mathcal{B}) \operatorname{var}_{a}^{b} F\right)\|g\|_{\infty} .
$$

(ii) [33, Proposition 11] Let $F \in(\mathcal{B}) B V([a, b], L(X))$ and $g_{n}:[a, b] \rightarrow X$ be such that the integral $\int_{a}^{b} \mathrm{~d}[F] g_{n}$ exists for all $n \in \mathbb{N}$ and $\lim _{n \rightarrow \infty}\left\|g_{n}-g\right\|_{\infty}=0$. Then the integral $\int_{a}^{b} \mathrm{~d}[F] g$ exists and

$$
\int_{a}^{b} \mathrm{~d}[F] g=\lim _{n \rightarrow \infty} \int_{a}^{b} \mathrm{~d}[F] g_{n}
$$

(iii) [33, Proposition 15] If $F \in(\mathcal{B}) B V([a, b], L(X)) \cap(\mathcal{B}) G([a, b], L(X))$ and $g \in G([a, b], X)$, then the integral $\int_{a}^{b} \mathrm{~d}[F]$ g exists.

(iv) [36, Theorem 13] If $F \in(\mathcal{B}) B V([a, b], L(X)) \cap G([a, b], L(X))$ and $g \in B V([a, b], X)$, then both integrals $\int_{a}^{b} F \mathrm{~d}[g]$ and $\int_{a}^{b} \mathrm{~d}[F]$ g exist, the sum

$$
\sum_{a \leq \tau<b} \Delta^{+} F(\tau) \Delta^{+} g(\tau)-\sum_{a<\tau \leq b} \Delta^{-} F(\tau) \Delta^{-} g(\tau)
$$

converges in $X$ and we have

$$
\left.\begin{array}{l}
\int_{a}^{b} F \mathrm{~d}[g]+\int_{a}^{b} \mathrm{~d}[F] g \\
\quad=F(b) g(b)-F(a) g(a)-\sum_{a \leq t<b} \Delta^{+} F(t) \Delta^{+} g(t)+\sum_{a<t \leq b} \Delta^{-} F(t) \Delta^{-} g(t)
\end{array}\right\}
$$

Remark 1.5. In the particular case when $F \in B V([a, b], L(X))$, the estimate (1.1) in Proposition 1.4 has a special form, namely,

$$
\left\|\int_{a}^{b} \mathrm{~d}[F] g\right\|_{X} \leq \int_{a}^{b} \mathrm{~d}\left[\operatorname{var}_{a}^{s} F\right]\|g(s)\|_{X} \leq \operatorname{var}_{a}^{b} F\|g\|_{\infty} .
$$

Besides the convergence result stated in Proposition 1.4 (ii), the next assertion provides another criteria which will be needed later. Since it corresponds to an obvious extension of Lemma 2.2 of [18] to Banach space-valued functions the proof was omitted.

Proposition 1.6. Let $g, g_{n} \in G([a, b], X)$ and $F, F_{n} \in B V([a, b], L(X))$ for all $n \in \mathbb{N}$. Assume

$$
\lim _{n \rightarrow \infty}\left\|g_{n}-g\right\|_{\infty}=0, \quad \lim _{n \rightarrow \infty}\left\|F_{n}-F\right\|_{\infty}=0 \quad \text { and } \quad \alpha^{*}:=\sup _{n \in \mathbb{N}} \operatorname{var}_{a}^{b} F_{n}<\infty
$$


Then

$$
\lim _{n \rightarrow \infty}\left(\sup _{t \in[a, b]}\left\|\int_{a}^{t} \mathrm{~d}\left[F_{n}\right] g_{n}-\int_{a}^{t} \mathrm{~d}[F] g\right\|_{X}\right)=0 .
$$

In the sequel, we prove some auxiliary properties of the $K S$-integral which are well-known in the finite dimensional case (cf. e.g. [43]), but are not available in the literature when involving the $\mathcal{B}$-variation of functions with values in a Banach space. Since the proofs are not straightforward adaptations from the results in finite dimension, we include them here.

Lemma 1.7. Let $F:[a, b] \rightarrow L(X)$ and $g:[a, b] \rightarrow X$.

(i) Let $F \in(\mathcal{B}) B V([a, b], L(X))$ and $g \in G([a, b], X)$. Then, for each partition $P$ of $[a, b]$, we have

$$
\|S(F, \mathrm{~d} g, P)\|_{X} \leq 2\|F\|_{S V}\|g\|_{\infty} .
$$

Moreover, if the integral $\int_{a}^{b} F \mathrm{~d}[g]$ exists, then

$$
\left\|\int_{a}^{b} F \mathrm{~d}[g]\right\|_{X} \leq 2\|F\|_{S V}\|g\|_{\infty} .
$$

(ii) Let $F \in G([a, b], L(X))$ and $g \in(\mathcal{B}) B V([a, b], X)$. Then, for each partition $P$ of $[a, b]$, we have

$$
\|S(\mathrm{~d} F, g, P)\|_{X} \leq 2\|F\|_{\infty}\|g\|_{S V} .
$$

Moreover, if the integral $\int_{a}^{b} \mathrm{~d}[F]$ g exists, then

$$
\left\|\int_{a}^{b} \mathrm{~d}[F] g\right\|_{X} \leq 2\|F\|_{\infty}\|g\|_{S V} .
$$

Proof. We will prove (i). The inequalities in (ii) can be obtained in a similar way.

Consider an arbitrary partition $P=\left(\xi_{j},\left[\alpha_{j-1}, \alpha_{j}\right]\right)$ of $[a, b]$ with $\nu(P)=m$. Note that

$$
\begin{aligned}
& S(F, \mathrm{~d} g, P) \\
&= F\left(\xi_{1}\right)\left[g\left(\alpha_{1}\right)-g(a)\right]+F\left(\xi_{2}\right)\left[g\left(\alpha_{2}\right)-g\left(\alpha_{1}\right)\right]+\ldots+F\left(\xi_{m}\right)\left[g(b)-g\left(\alpha_{m-1}\right)\right] \\
&= F(b) g(b)-F(a) g(a) \\
&-\left[F\left(\xi_{1}\right)-F(a)\right] g(a)-\left[F\left(\xi_{2}\right)-g\left(\xi_{1}\right)\right]-\ldots-\left[F(b)-F\left(\xi_{m}\right)\right] g(b) \\
&= F(b) g(b)-F(a) g(a)-\sum_{j=0}^{m}\left[F\left(\xi_{j+1}\right)-F\left(\xi_{j}\right)\right] g\left(\alpha_{j}\right),
\end{aligned}
$$


where $\xi_{0}=a$ and $\xi_{m+1}=b$. Consequently, by the fact that $F \in(\mathcal{B}) B V([a, b], L(X))$,

$$
\begin{aligned}
\|S(F, \mathrm{~d} g, P)\|_{X} \leq & \left(\|F(a)\|_{L(X)}+\|F(b)\|_{L(X)}\right)\|g\|_{\infty} \\
& +\left\|\sum_{j=0}^{m}\left[F\left(\xi_{j+1}\right)-F\left(\xi_{j}\right)\right] \frac{g\left(\alpha_{i}\right)}{\left\|g\left(\alpha_{i}\right)\right\|_{X}}\right\| g\left(\alpha_{i}\right)\left\|_{X}\right\|_{X} \\
\leq & \left(\|F(a)\|_{L(X)}+\|F(b)\|_{L(X)}+\left\|\sum_{j=0}^{m}\left[F\left(\xi_{j+1}\right)-F\left(\xi_{j}\right)\right] \frac{g\left(\alpha_{i}\right)}{\left\|g\left(\alpha_{i}\right)\right\|_{X}}\right\|_{X}\right)\|g\|_{\infty} \\
\leq & \left(\|F(a)\|_{L(X)}+\|F(b)\|_{L(X)}+(\mathcal{B}) \operatorname{var}_{a}^{b} F\right)\|g\|_{\infty} \leq 2\|F\|_{S V}\|g\|_{\infty},
\end{aligned}
$$

and (1.4) holds.

Now, assuming that the integral $\int_{a}^{b} F \mathrm{~d}[g]$ exists, given $\varepsilon>0$, there is a gauge $\delta$ on $[a, b]$ such that

$$
\left\|S(F, \mathrm{~d} g, P)-\int_{a}^{b} F \mathrm{~d}[g]\right\|_{X}<\varepsilon, \quad \text { whenever } P \text { is } \delta \text {-fine partition of }[a, b] .
$$

Fixing a $\delta$-fine partition $P_{\varepsilon}$ of $[a, b]$, by (1.4), we have

$$
\begin{aligned}
\left\|\int_{a}^{b} F \mathrm{~d}[g]\right\|_{X} & \leq\left\|\int_{a}^{b} F \mathrm{~d}[g]-S\left(F, \mathrm{~d} g, P_{\varepsilon}\right)\right\|_{X}+\left\|S\left(F, \mathrm{~d} g, P_{\varepsilon}\right)\right\|_{X} \\
& <\varepsilon+2\|F\|_{S V}\|g\|_{\infty}
\end{aligned}
$$

Since $\varepsilon>0$ is arbitrary, inequality (1.5) is true.

Lemma 1.8. Let $F:[a, b] \rightarrow L(X)$ be a finite step function. Then for any $g:[a, b] \rightarrow X$ the integral $\int_{a}^{b} \mathrm{~d}[F]$ g exists. Symmetrically, if $g:[a, b] \rightarrow X$ is a finite step function, then $\int_{a}^{b} F \mathrm{~d}[g]$ exists for any $F:[a, b] \rightarrow L(X)$.

Proof. We will only prove the case when $F$ is a finite step function and $g:[a, b] \rightarrow X$ is an arbitrary function. The second possibility can be shown similarly.

Notice that, $F$ can be written as

$$
F=\sum_{j=1}^{m} \chi_{\left(\alpha_{j-1}, \alpha_{j}\right)} \widetilde{T}_{j}+\sum_{j=0}^{m} \chi_{\left[\alpha_{j}\right]} F\left(\alpha_{j}\right),
$$

for some division $D=\left\{\alpha_{0}, \alpha_{1}, \ldots, \alpha_{m}\right\}$ of $[a, b]$ and $\widetilde{T}_{j} \in L(X), j=1, \ldots, m$. Hence, by the linearity of the integral, it is sufficient to prove the existence of the integral for functions of the 
form

$$
\chi_{(c, d)} \widetilde{T} \quad \text { and } \quad \chi_{[c]} \widetilde{S}, \quad \text { with } c, d \in[a, b], \widetilde{T}, \widetilde{S} \in L(X)
$$

Assume $F=\chi_{(c, d)} \widetilde{T}$. Given $\varepsilon>0$, define

$$
\delta(t)=\left\{\begin{array}{cl}
\varepsilon, & \text { if } t=c \text { or } t=d \\
\frac{1}{2} \min \{|t-c|,|t-d|\}, & \text { if } t \in[a, b] \backslash\{c, d\}
\end{array} .\right.
$$

For any $\delta$-fine partition $P$ of $[a, b], c$ and $d$ are necessarily tags and $S(\mathrm{~d} F, g, P)=\widetilde{T}[g(c)-g(d)]$. Thus

$$
\int_{a}^{b} \mathrm{~d}[F] g=\widetilde{T}[g(c)-g(d)]
$$

The proof for the case $F=\chi_{[d]} \widetilde{S}$ is analogous.

Besides the Bolzano-Cauchy criteria for existence of the $K S$-integral (see [33, Proposition 7]), Š. Schwabik provided, in the same paper, another condition under which such integral exists (see Proposition 1.4 (iii)). The next theorem is our first outcome and supplements Schwabik's result.

Theorem 1.9. Let $F:[a, b] \rightarrow L(X)$ and $g:[a, b] \rightarrow X$.

(i) If $F \in G([a, b], L(X))$ and $g \in(\mathcal{B}) B V([a, b], X)$, then the integral $\int_{a}^{b} \mathrm{~d}[F] g$ exists.

(ii) If $F \in(\mathcal{B}) B V([a, b], L(X))$ and $g \in G([a, b], X)$, then the integral $\int_{a}^{b} F \mathrm{~d}[g]$ exists.

Proof. We will prove assertion (i). The second assertion can be proved with similar arguments.

By Proposition 1.1 (i), there exists a sequence of finite step functions $F_{n}:[a, b] \rightarrow L(X), n \in \mathbb{N}$, such that $\lim _{n \rightarrow \infty}\left\|F_{n}-F\right\|_{\infty}=0$.

By Lemma 1.8, the integral $\int_{a}^{b} \mathrm{~d}\left[F_{n}\right] g$ exists, for each $n \in \mathbb{N}$. Moreover, these integrals define a Cauchy sequence in the Banach space $X$. Indeed, given $\varepsilon>0$, there is $n_{0} \in \mathbb{N}$ such that $\left\|F_{n}-F\right\|_{\infty}<\varepsilon$, for $n \geq n_{0}$. Thus, using (1.7), we obtain

$$
\left\|\int_{a}^{b} \mathrm{~d}\left[F_{n}-F_{m}\right] g\right\|_{X} \leq 2\left\|F_{n}-F_{m}\right\|_{\infty}\|g\|_{S V} \leq 4 \varepsilon\|g\|_{S V}, \quad \text { for all } m, n \geq n_{0} .
$$


Let $I \in X$ be such that $I=\lim _{n \rightarrow \infty} \int_{a}^{b} \mathrm{~d}\left[F_{n}\right] g$. Hence, there is $N \in \mathbb{N}$, with $N \geq n_{0}$, such that

$$
\left\|\int_{a}^{b} \mathrm{~d}\left[F_{N}\right] g-I\right\|_{X}<\varepsilon
$$

Assume $\delta$ is a gauge on $[a, b]$ such that

$$
\left\|S\left(\mathrm{~d} F_{N}, g, P\right)-\int_{a}^{b} \mathrm{~d}\left[F_{N}\right] g\right\|_{X}<\varepsilon, \quad \text { whenever } P \text { is a } \delta \text {-fine partition of }[a, b] .
$$

Having this in mind together with (1.6), let us show that $I$ is the $K S$-integral of $g$ with respect to $F$ on $[a, b]$. For an arbitrary $\delta$-fine partition $P$ of $[a, b]$, we get

$$
\begin{aligned}
\| S(\mathrm{~d} F, & g, P)-I \|_{X} \\
\leq & \left\|S(\mathrm{~d} F, g, P)-S\left(\mathrm{~d} F_{N}, g, P\right)\right\|_{X}+\left\|S\left(\mathrm{~d} F_{N}, g, P\right)-\int_{a}^{b} \mathrm{~d}\left[F_{N}\right] g\right\|_{X} \\
& +\left\|\int_{a}^{b} \mathrm{~d}\left[F_{N}\right] g-I\right\|_{X} \\
& <2\left\|F-F_{N}\right\|_{\infty}\|g\|_{S V}+2 \varepsilon<2 \varepsilon\left(\|g\|_{S V}+1\right),
\end{aligned}
$$

which concludes the proof of assertion (i).

A direct consequence of Theorem 1.9 combined with Lemma 1.7 follows next.

Corollary 1.10. The following assertions hold:

(i) Let $F, F_{n} \in G([a, b], L(X)), n \in \mathbb{N}$, be such that $\lim _{n \rightarrow \infty}\left\|F_{n}-F\right\|_{\infty}=0$. Then, for any $g \in(\mathcal{B}) B V([a, b], X)$, the integrals

$$
\int_{a}^{b} \mathrm{~d}[F] g \quad \text { and } \quad \int_{a}^{b} \mathrm{~d}\left[F_{n}\right] g, n \in \mathbb{N},
$$

exist and

$$
\lim _{n \rightarrow \infty} \int_{a}^{b} \mathrm{~d}\left[F_{n}\right] g=\int_{a}^{b} \mathrm{~d}[F] g .
$$

(ii) Let $g, g_{n} \in G([a, b], X), n \in \mathbb{N}$, be such that $\lim _{n \rightarrow \infty}\left\|g_{n}-g\right\|_{\infty}=0$. Then, for any function $F \in(\mathcal{B}) B V([a, b], L(X))$, the integrals

$$
\int_{a}^{b} F \mathrm{~d}[g] \text { and } \int_{a}^{b} F \mathrm{~d}\left[g_{n}\right], n \in \mathbb{N}
$$


exist and

$$
\lim _{n \rightarrow \infty} \int_{a}^{b} F \mathrm{~d} g_{n}=\int_{a}^{b} F \mathrm{~d} g
$$

Using Corollary 1.10, we are now able to present a general form of the integration by parts theorem proposed by Š. Schwabik in [36]. Nevertheless, we need an auxiliar estimate to the series in (1.2).

Lemma 1.11. Let $F:[a, b] \rightarrow L(X)$ and $g:[a, b] \rightarrow X$.

(i) If $F \in(\mathcal{B}) B V([a, b], L(X)) \cap G([a, b], L(X))$ and $g \in G([a, b], X)$, then

$$
\left\|\sum_{t \in[a, b)} \Delta^{+} F(t) \Delta^{+} g(t)\right\|_{X}+\left\|\sum_{t \in(a, b]} \Delta^{-} F(t) \Delta^{-} g(t)\right\|_{X} \leq 4\left((\mathcal{B}) \operatorname{var}_{a}^{b} F\right)\|g\|_{\infty} .
$$

(ii) If $F \in G([a, b], L(X))$ and $g \in(\mathcal{B}) B V([a, b], X) \cap G([a, b], X)$, then

$$
\left\|\sum_{t \in[a, b)} \Delta^{+} F(t) \Delta^{+} g(t)\right\|_{X}+\left\|\sum_{t \in(a, b]} \Delta^{-} F(t) \Delta^{-} g(t)\right\|_{X} \leq 4\|F\|_{\infty}\left((\mathcal{B}) \operatorname{var}_{a}^{b} g\right) .
$$

Proof. Assuming $F \in(\mathcal{B}) B V([a, b], L(X)) \cap G([a, b], L(X))$ and $g \in G([a, b], X)$, by [36, Lemma 11], the series in (1.8) converge. Let us prove that

$$
\left\|\sum_{t \in[a, b)} \Delta^{+} F(t) \Delta^{+} g(t)\right\|_{X} \leq 2\left((\mathcal{B}) \operatorname{var}_{a}^{b} F\right)\|g\|_{\infty}
$$

Consider $\left\{t_{k} \in X ; k \in \mathbb{N}\right\}$ the set of common points of discontinuity of the functions $F$ and $g$ on $(a, b)$, which by Proposition 1.1 (ii) is at most countable. Without loss of generality, we may assume $t_{k}<t_{k+1}$, for every $k \in \mathbb{N}$.

For each $n \in \mathbb{N}$, define

$$
S_{n}=\Delta^{+} F(a) \Delta^{+} g(a)+\sum_{k=1}^{n} \Delta^{+} F\left(t_{k}\right) \Delta^{+} g\left(t_{k}\right) .
$$

Given $\varepsilon>0$ and $n \in \mathbb{N}$, since $F$ is regulated, for each $k=1,2, \ldots, n$, choose $\delta_{k}>0$ in such a way that $\left\|F\left(t_{k}+\right)-F\left(t_{k}+\delta_{k}\right)-\right\|_{L(X)}<\frac{\varepsilon}{8(n+1)\|g\|_{\infty}} \quad$ and $\quad\left[t_{k}-\delta_{k}, t_{k}+\delta_{k}\right] \cap\left\{t_{1}, t_{2}, \ldots, t_{n}\right\}=\left\{t_{k}\right\}$. 
Analogously, let $\delta_{0}>0$ be such that $b-\delta_{0}>t_{n}$ and also

$$
\left\|F(a+)-F\left(a+\delta_{0}\right)\right\|_{L(X)}<\frac{\varepsilon}{8\|g\|_{\infty}} \quad \text { and } \quad a+\delta_{0}<t_{1}
$$

Using these inequalities and since $\left\|\Delta^{+} g(t)\right\|_{X} \leq 2\|g\|_{\infty}$, for $t \in[a, b)$, we obtain

$$
\begin{aligned}
\left\|S_{n}\right\|_{X}<2 & \|g\|_{\infty}\left(\left\|F(a+)-F\left(a+\delta_{0}\right)\right\|_{L(X)}+\sum_{k=1}^{n}\left\|F\left(t_{k}+\right)-F\left(t_{k}+\delta_{k}\right)\right\|_{L(X)}\right) \\
& +\left\|\left[F\left(a+\delta_{0}\right)-F(a)\right] \Delta^{+} g(a)+\sum_{k=1}^{n}\left[F\left(t_{k}+\delta_{k}\right)-F\left(t_{k}\right)\right] \Delta^{+} g\left(t_{k}\right)\right\|_{X} \\
< & \frac{\varepsilon}{4}+\frac{n \varepsilon}{4(n+1)} \\
& +2\|g\|_{\infty}\left\|\left[F\left(a+\delta_{0}\right)-F(a)\right] \frac{\Delta^{+} g(a)}{2\|g\|_{\infty}}+\sum_{k=1}^{n}\left[F\left(t_{k}+\delta_{k}\right)-F\left(t_{k}\right)\right] \frac{\Delta^{+} g\left(t_{k}\right)}{2\|g\|_{\infty}}\right\|_{X}
\end{aligned}
$$

Therefore

$$
\begin{aligned}
\left\|S_{n}\right\|_{X} & <\varepsilon+2\|g\|_{\infty}\left\|\left[F\left(a+\delta_{0}\right)-F(a)\right] \frac{\Delta^{+} g(a)}{2\|g\|_{\infty}}+\sum_{k=1}^{n}\left[F\left(t_{k}+\delta_{k}\right)-F\left(t_{k}\right)\right] \frac{\Delta^{+} g\left(t_{k}\right)}{2\|g\|_{\infty}}\right\|_{X} \\
& \leq \varepsilon+2\|g\|_{\infty}\left((\mathcal{B}) \operatorname{var}_{a}^{b} F\right) .
\end{aligned}
$$

Since $\varepsilon>0$ is arbitrary, we get

$$
\left\|S_{n}\right\|_{X} \leq 2\|g\|_{\infty}\left((\mathcal{B}) \operatorname{var}_{a}^{b} F\right)
$$

which, together with the fact that

$$
\sum_{t \in[a, b)} \Delta^{+} F(t) \Delta^{+} g(t)=\lim _{n \rightarrow \infty} S_{n}
$$

lead to (1.9). Analogously, we can estimate the sum $\sum_{t \in(a, b]} \Delta^{-} F(t) \Delta^{-} g(t)$, which concludes the proof of (i).

In case $F \in G([a, b], L(X))$ and $g \in(\mathcal{B}) B V([a, b], X) \cap G([a, b], X)$, we can proceed similarly. 
Corollary 1.12 (Integration by Parts). If $F \in(\mathcal{B}) B V([a, b], L(X)) \cap G([a, b], L(X))$ and $g \in$ $G([a, b], X)$ or $F \in G([a, b], L(X))$ and $g \in(\mathcal{B}) B V([a, b], X) \cap G([a, b], X)$, then both integrals

$$
\int_{a}^{b} F \mathrm{~d}[g] \text { and } \int_{a}^{b} \mathrm{~d}[F] g
$$

exist and (1.3) holds, that is,

$$
\left.\begin{array}{l}
\int_{a}^{b} F \mathrm{~d}[g]+\int_{a}^{b} \mathrm{~d}[F] g \\
\quad=F(b) g(b)-F(a) g(a)-\sum_{a \leq t<b} \Delta^{+} F(t) \Delta^{+} g(t)+\sum_{a<t \leq b} \Delta^{-} F(t) \Delta^{-} g(t) .
\end{array}\right\}
$$

Proof. Suppose $F \in(\mathcal{B}) B V([a, b], L(X)) \cap G([a, b], L(X))$ and $g \in G([a, b], X)$. By Proposition 1.1 (i), there is a sequence $\left\{g_{n}\right\}$ of finite step functions on $[a, b]$ which tends uniformly to $g$ on $[a, b]$. Since $g_{n} \in B V([a, b], X)$, for each $n \in \mathbb{N}$, by Proposition 1.4 (iv), we have

$$
\left.\begin{array}{l}
\int_{a}^{b} F \mathrm{~d}\left[g_{n}\right]+\int_{a}^{b} \mathrm{~d}[F] g_{n} \\
\quad=F(b) g_{n}(b)-F(a) g_{n}(a)-\sum_{a \leq t<b} \Delta^{+} F(t) \Delta^{+} g_{n}(t)+\sum_{a<t \leq b} \Delta^{-} F(t) \Delta^{-} g_{n}(t)
\end{array}\right\}
$$

Using (1.8), we get

$$
\begin{gathered}
\| \sum_{a \leq t<b} \Delta^{+} F(t) \Delta^{+}(g(t) \\
\left.-g_{n}(t)\right)-\sum_{a<t \leq b} \Delta^{-} F(t) \Delta^{-}\left(g(t)-g_{n}(t)\right) \|_{X} \\
\leq 4\left((\mathcal{B}) \operatorname{var}_{a}^{b} F\right)\left\|g-g_{n}\right\|_{\infty}
\end{gathered}
$$

and consequently, by the fact that $\lim _{n \rightarrow \infty}\left\|g_{n}-g\right\|_{\infty}=0$, we obtain

$$
\begin{gathered}
\lim _{n \rightarrow \infty}\left(\sum_{a \leq t<b} \Delta^{+} F(t) \Delta^{+} g_{n}(t)-\sum_{a<t \leq b} \Delta^{-} F(t) \Delta^{-} g_{n}(t)\right) \\
=\sum_{a \leq t<b} \Delta^{+} F(t) \Delta^{+} g(t)-\sum_{a<t \leq b} \Delta^{-} F(t) \Delta^{-} g(t) .
\end{gathered}
$$

On the other hand, we have

$$
\begin{aligned}
\lim _{n \rightarrow \infty}\left(\int_{a}^{b} F \mathrm{~d}\left[g_{n}\right]+\int_{a}^{b} \mathrm{~d}[F] g_{n}-F(b) g_{n}(b)+F(a) g_{n}(a)\right) \\
=\int_{a}^{b} F \mathrm{~d}[g]+\int_{a}^{b} \mathrm{~d}[F] g-F(b) g(b)+F(a) g(a) .
\end{aligned}
$$


In summary, letting $n \rightarrow \infty$ in (1.10), we obtain (1.3).

The second possibility, when $F \in G([a, b], L(X))$ and $g \in(\mathcal{B}) B V([a, b], X) \cap G([a, b], X)$, can be proved in a similar way.

In [28], results on Integration by Parts for some generalizations of the Riemman-Stieltjes integral are discussed. In particular, using a different approach than the one presented here, K. M. Naralenkov gives necessary and sufficient conditions for such type of results for the socalled Henstock-Stieltjes integral in a Banach space-valued setting.

We will close this section by formulating results on integration by substitution for the $K S$ integral. Substitution formulas for vector integrals were the object of the Master Dissertation of M. Federson [6] (see also [7]). Among the results of such study, it is worth mentioning the following one.

\section{Proposition 1.13.}

(i) [7, Theorem 11] Let $F:[a, b] \rightarrow L(X)$ and $g:[a, b] \rightarrow X$ be such that the integral $\int_{a}^{b} F \mathrm{~d}[g]$ exists. Given $H \in(\mathcal{B}) B V([a, b], L(X))$, if at least one of the integrals

$$
\int_{a}^{b} H(t) \mathrm{d}_{t}\left[\int_{a}^{t} F \mathrm{~d}[g]\right], \quad \int_{a}^{b} H F \mathrm{~d}[g]
$$

exists, then the other one also exists and

$$
\int_{a}^{b} H(t) \mathrm{d}_{t}\left[\int_{a}^{t} F \mathrm{~d}[g]\right]=\int_{a}^{b} H F \mathrm{~d}[g] .
$$

(ii) [7, Theorem 12] Let $F, H:[a, b] \rightarrow L(X)$ be such that the integral $\int_{a}^{b} \mathrm{~d}[F] H$ exists. Given $g \in B V([a, b], X)$, if at least one of the integrals

$$
\int_{a}^{b} \mathrm{~d}_{t}\left[\int_{a}^{t} \mathrm{~d}[F] H\right] g(t), \quad \int_{a}^{b} \mathrm{~d}[F] H g
$$

exists, then the other one also exists and

$$
\int_{a}^{b} \mathrm{~d}_{t}\left[\int_{a}^{t} \mathrm{~d}[F] H\right] g(t)=\int_{a}^{b} \mathrm{~d}[F] H g .
$$


In the next theorem we give a substituion formula for the case when an integral of the type $\int_{a}^{b} H \mathrm{~d}[F] g$ appears. The proof follows closely the ideas presented by M. Federson in [7].

Theorem 1.14 (Substitution Theorem). Let $F:[a, b] \rightarrow L(X)$ and $g:[a, b] \rightarrow X$ be such that the integral $\int_{a}^{b} \mathrm{~d}[F]$ g exists. Given a function $H \in(\mathcal{B}) B V([a, b], L(X))$, if at least one of the integrals

exists, then the other one also exists and

$$
\int_{a}^{b} H(t) \mathrm{d}_{t}\left[\int_{a}^{t} \mathrm{~d}[F] g\right], \quad \int_{a}^{b} H \mathrm{~d}[F] g
$$

$$
\int_{a}^{b} H(t) \mathrm{d}_{t}\left[\int_{a}^{t} \mathrm{~d}[F] g\right]=\int_{a}^{b} H \mathrm{~d}[F] g
$$

Proof. Let us assume that the integral $\int_{a}^{b} H \mathrm{~d}[F] g$ exists.

Given $\varepsilon>0$, let $\delta_{1}$ and $\delta_{2}$ be gauges on $[a, b]$ such that

$$
\left\|S(H, \mathrm{~d} F, g, P)-\int_{a}^{b} H \mathrm{~d}[F] g\right\|_{X}<\varepsilon \text { for all } \delta_{1} \text {-fine partitions } P \text { of }[a, b]
$$

and

$$
\left\|S(\mathrm{~d} F, g, P)-\int_{a}^{b} \mathrm{~d}[F] g\right\|_{X}<\varepsilon \text { for all } \delta_{2} \text {-fine partitions } P \text { of }[a, b] .
$$

The Saks-Henstock Lemma (see [33, Lemma 16]) states that, for any $\delta_{2}$-fine partition of $[a, b]$, $P=\left(\xi_{j},\left[\alpha_{j-1}, \alpha_{j}\right]\right)$, we have

$$
\left\|\sum_{k=j}^{\nu(P)}\left[F\left(\alpha_{k}\right)-F\left(\alpha_{k-1}\right)\right] g\left(\xi_{k}\right)-\int_{\alpha_{k-1}}^{\alpha_{k}} \mathrm{~d}[F] g\right\|_{X} \leq \varepsilon \quad \text { for } j=1,2, \ldots, \nu(P) .
$$

Define $K(t)=\int_{a}^{t} \mathrm{~d}[F] g$, for $t \in[a, b]$.

If $P=\left(\xi_{j},\left[\alpha_{j-1}, \alpha_{j}\right]\right)$ is a $\delta$-fine partition of $[a, b]$, where $\delta(t)=\min \left\{\delta_{1}(t), \delta_{2}(t)\right\}, t \in[a, b]$, then by (1.12), we get

$$
\begin{aligned}
\left\|S(H, \mathrm{~d} K, P)-\int_{a}^{b} H \mathrm{~d}[F] g\right\|_{X} \\
\quad \leq\|S(H, \mathrm{~d} K, P)-S(H \mathrm{~d} F, g, P)\|_{X}+\left\|S(H, \mathrm{~d} F, g, P)-\int_{a}^{b} H \mathrm{~d}[F] g\right\|_{X} \\
\quad<\left\|\sum_{j=1}^{\nu(P)} H\left(\xi_{j}\right)\left(\int_{\alpha_{j-1}}^{\alpha_{j}} \mathrm{~d}[F] g-\left[F\left(\alpha_{j}\right)-F\left(\alpha_{j-1}\right)\right] g\left(\xi_{j}\right)\right)\right\|_{X}+\varepsilon
\end{aligned}
$$


As noticed in the proof of Theorem 11 in [7], the following equality holds

$$
\sum_{j=1}^{m} A_{j} x_{j}=\sum_{j=1}^{m}\left[A_{j}-A_{j-1}\right]\left(\sum_{k=j}^{m} x_{k}\right)+A_{0}\left(\sum_{k=1}^{m} x_{k}\right)
$$

for all $A_{j} \in L(X)$ and all $x_{j} \in X$. Taking $m=\nu(P)$ and also

$$
A_{j}=H\left(\xi_{j}\right), \quad A_{0}=H(a), \quad x_{j}=\left[F\left(\alpha_{j}\right)-F\left(\alpha_{k-1}\right)\right] g\left(\xi_{j}\right)-\int_{\alpha_{j-1}}^{\alpha_{j}} \mathrm{~d}[F] g,
$$

for $j=1, \ldots \nu(P)$, by (1.13), we obtain

$$
\begin{aligned}
& \left\|S(H, \mathrm{~d} K, P)-\int_{a}^{b} H \mathrm{~d}[F] g\right\|_{X} \\
& <\varepsilon+\left\|\sum_{j=1}^{\nu(P)}\left[H\left(\xi_{j}\right)-H\left(\xi_{j-1}\right)\right]\left(\sum_{k=j}^{\nu(P)} x_{k}\right)\right\|_{X} \\
& +\left\|H(a)\left(\sum_{k=1}^{m}\left[F\left(\alpha_{k}\right)-F\left(\alpha_{k-1}\right)\right] g\left(\xi_{k}\right)-\int_{\alpha_{k-1}}^{\alpha_{k}} \mathrm{~d}[F] g\right)\right\|_{X} \\
& \quad\left\|\varepsilon+\sum_{j=1}\left[H\left(\xi_{j}\right)-H\left(\xi_{j-1}\right)\right] \frac{\sum_{k=j}^{\nu(P)} x_{k}}{\varepsilon}\right\|_{X} \\
& +\|H(a)\|_{L(X)}\left\|\sum_{k=1}^{\nu(P)}\left[F\left(\alpha_{k}\right)-F\left(\alpha_{k-1}\right)\right] g\left(\xi_{k}\right)-\int_{\alpha_{k-1}}^{\alpha_{k}} \mathrm{~d}[F] g\right\|_{X} \\
& <\varepsilon\left(1+(\mathcal{B}) \operatorname{var}_{a}^{b} H+\|H(a)\|_{X}\right) .
\end{aligned}
$$

Hence the integral $\int_{a}^{b} H \mathrm{~d}[K]$ exists and (1.11) holds.

The other implication can be shown in a similar way.

The last result of this chapter provides a different substitution formula not covered by previous theorem, by assuming $H \in G([a, b], L(X))$ instead of $H \in(\mathcal{B}) B V([a, b], L(X))$. On the other hand, stronger conditions over $F$ and $g$ are imposed.

Unlike Theorem 1.14, the proof of the our second substitution theorem does not rely on the Saks-Henstock Lemma, but on a convergence argument. To this aim, the following assertion will be needed. 
Proposition 1.15. Let $F \in B V([a, b], L(X)), H_{n}:[a, b] \rightarrow L(X)$ and $g:[a, b] \rightarrow X$ be such that the integral $\int_{a}^{b} H_{n} \mathrm{~d}[F]$ g exists for all $n \in \mathbb{N}$. If $g$ is a bounded function and $\lim _{n \rightarrow \infty}\left\|H_{n}-H\right\|_{\infty}=0$, then the integral $\int_{a}^{b} H \mathrm{~d}[F] g$ exists and

$$
\lim _{n \rightarrow \infty}\left\|\int_{a}^{b} H_{n} \mathrm{~d}[F] g-\int_{a}^{b} H \mathrm{~d}[F] g\right\|_{X}=0
$$

Proof. It follows from the same argument used in [33, Proposition 11].

Theorem 1.16 (Second Substitution Theorem). Let $F \in B V([a, b], L(X))$ and $g:[a, b] \rightarrow X$, bounded, be such that the integral $\int_{a}^{b} \mathrm{~d}[F]$ g exists. Then, for each $H \in G([a, b], L(X))$, both integrals in (1.11) exist and equality (1.11) holds.

Proof. Step 1. At first, we show that (1.11) holds when $H:[a, b] \rightarrow L(X)$ is a finite step function, which means that $H$ can be written as linear combination of functions of the form

$$
\chi_{[a, \tau]}(t) \widetilde{H}_{1}, \chi_{[\sigma, b]}(t) \widetilde{H}_{2}, \chi_{[a]}(t) \widetilde{H}_{3}, \chi_{[b]}(t) \widetilde{H}_{4},
$$

where $\tau, \sigma \in(a, b)$ and $\widetilde{H}_{i} \in L(X) i=1,2,3,4$. By the linearity of the integral, it is enough to prove (1.11) for such functions.

Assume $H=\chi_{[a, \tau]} \widetilde{H}$, for $\tau \in(a, b), \widetilde{H} \in L(X)$, and put $K(t)=\int_{a}^{t} \mathrm{~d}[F] g$, for $t \in[a, b]$. Obviously,

$$
\int_{a}^{\tau} H \mathrm{~d}[F] g=\int_{a}^{\tau} H \mathrm{~d}[K]=\widetilde{H} \int_{a}^{\tau} \mathrm{d}[F] g
$$

Given $\varepsilon>0$, let

$$
\delta(t)=\left\{\begin{array}{cl}
\varepsilon, & \text { if } t=\tau, \\
\frac{1}{2}|\tau-t|, & \text { if } \tau<t \leq b .
\end{array}\right.
$$

Then, for any $\delta$-fine partition $P$ of $[\tau, b]$, with $D=\left\{\alpha_{0}, \alpha_{1}, \ldots, \alpha_{m}\right\}$ and $\xi=\left(\xi_{1}, \xi_{2} \ldots, \xi_{m}\right)$, we have $\xi_{1}=\alpha_{0}=\tau, \alpha_{1}<\tau+\varepsilon$, and moreover

$$
S(H, \mathrm{~d} F, g, P)=\widetilde{H}\left[F\left(\alpha_{1}\right)-F(\tau)\right] g(\tau) \quad \text { and } \quad S(H, \mathrm{~d} K, P)=\widetilde{H}\left[K\left(\alpha_{1}\right)-K(\tau)\right]
$$


As a consequence of the Hake's Theorem for the $K S$-integral (see [33, Corollary 24]), we get

$$
\int_{\tau}^{b} H \mathrm{~d}[F] g=\widetilde{H} \Delta^{+} F(\tau) g(\tau) \quad \text { and } \quad \int_{\tau}^{b} H \mathrm{~d}[K]=\widetilde{H} \Delta^{+} K(\tau)=\widetilde{H} \Delta^{+} F(\tau) g(\tau),
$$

that is,

$$
\int_{\tau}^{b} H \mathrm{~d}[F] g=\int_{\tau}^{b} H \mathrm{~d}[K]=\widetilde{H} \Delta^{+} F(\tau) g(\tau) .
$$

This together with (1.14) yield (1.11).

The remaining cases $H=\chi_{[\tau, b]} \widetilde{H}, H=\chi_{[a]} \widetilde{H}$ and $H=\chi_{[b]} \widetilde{H}$ have similar proofs.

Step 2. Let $H \in G([a, b], L(X))$ and consider the sequence $H_{n}:[a, b] \rightarrow L(X), n \in \mathbb{N}$, of finite step functions such that $\lim _{n \rightarrow \infty}\left\|H_{n}-H\right\|_{\infty}=0$.

Put, again, $K(t)=\int_{a}^{t} \mathrm{~d}[F] g$, for $t \in[a, b]$. By step 1 and Proposition 1.15, we have

$$
\lim _{n \rightarrow \infty} \int_{a}^{b} H_{n} \mathrm{~d}[K]=\lim _{n \rightarrow \infty} \int_{a}^{b} H_{n} \mathrm{~d}[F] g=\int_{a}^{b} H \mathrm{~d}[F] g .
$$

On the other hand, noticing that $K \in B V([a, b], L(X))$, by Proposition 1.4 (ii), it follows that

$$
\lim _{n \rightarrow \infty} \int_{a}^{b} H_{n} \mathrm{~d}[K]=\int_{a}^{b} H \mathrm{~d}[K]=\int_{a}^{b} H(t) \mathrm{d}_{t}\left[\int_{a}^{t} \mathrm{~d}[F] g\right]
$$

which leads to (1.11). 


$\frac{10}{2}$

\section{Generalized linear differential equations in Banach space}

In the present chapter, we introduce the main object of our studies, namely, linear integral equations of the form

$$
x(t)=\widetilde{x}+\int_{a}^{t} \mathrm{~d}[A] x+f(t)-f(a), \quad t \in[a, b] .
$$

with respect to the Kurzweil-Stieltjes integral for Banach space-valued functions.

This chapter is divided into three sections. The first one presents some basic results on linear generalized ODEs (c.f. [35] and [36]) and adds some auxiliar assertions which are well-known in the finite dimensional case (see [43]). The second section brings up our theorem on continuous dependence of solutions on parameters which was inspired by the classical Z. Opial's result (see [30]). In addition, using an assumption of uniformly bounded variation, in the third section, we present a result on continuous dependence on parameters for linear nonhomogeneous equations. 


\subsection{Basic results}

Let $A \in B V([a, b], L(X)), f \in G([a, b], X)$ and $\widetilde{x} \in X$, where $X$ is a fixed Banach space. Consider the nonhomogeneous integral equation

$$
x(t)=\widetilde{x}+\int_{a}^{t} \mathrm{~d}[A] x+f(t)-f(a), \quad t \in[a, b],
$$

where the Stieltjes-type integral is in the sense of Kurzweil (see Chapter 1).

The equation $(\mathrm{NH})$ is called here generalized linear integral equation. In case $X$ is the Euclidean space, a complete theory can be found, for instance, in the monographs by Š. Schwabik [32] and by Š. Schwabik, M. Tvrdý and O. Vejvoda [38].

It is worth mentioning that, using the symbolical notation introduced by J. Kurzweil in [24], the linear problem $(\mathrm{NH})$ corresponds to the generalized ordinary differential equation

$$
\frac{\mathrm{d} x}{\mathrm{~d} \tau}=D[A(t) x+f(t)], \quad x(a)=\widetilde{x}
$$

whose definition is given by the solutions of (NH). Indeed, a function $x:[a, b] \rightarrow X$ is a solution of (2.1) on $[a, b]$, if the $K S$-integral $\int_{a}^{b} \mathrm{~d}[A] x$ exists and $x$ satisfies the equality (NH) for each $t \in[a, b]$. Clearly $x(a)=\widetilde{x}$ whenever $x$ is a solution of $(\mathrm{NH})$.

Fundamental results on the existence and uniqueness of a solution of (NH) were established by Š. Schwabik in [34]. In particular, taking into account the closing remark in [34], the following result is a special case of Proposition 2.8 from [34].

Proposition 2.1. Let $A \in B V([a, b], L(X))$ be such that

$$
\left[I-\Delta^{-} A(t)\right]^{-1} \in L(X), \quad \text { for all } t \in(a, b]
$$

Then, for every $\widetilde{x} \in X$ and every $f \in G([a, b], X)$, the equation (NH) has a unique solution $x$ on $[a, b]$ and $x \in G([a, b], X)$.

On the solutions of $(\mathrm{NH})$ the following assertions are true. 
Lemma 2.2. Let $A \in B V([a, b], L(X))$ satisfy (E), $f \in G([a, b], X)$ and $\widetilde{x} \in X$. If $x$ is the corresponding solution of $(\mathrm{NH})$ on $[a, b]$, we have

$$
\begin{gathered}
\Delta^{+} x(t)=\Delta^{+} A(t) x(t)+\Delta^{+} f(t) \quad \text { for } t \in[a, b), \\
\Delta^{-} x(t)=\Delta^{-} A(t) x(t)+\Delta^{-} f(t) \quad \text { for } t \in(a, b], \\
(x-f) \in B V([a, b], X) \quad \text { with } \quad \operatorname{var}_{a}^{b}(x-f) \leq\left(\operatorname{var}_{a}^{b} A\right)\|x\|_{\infty}, \\
c_{A}:=\sup \left\{\left\|\left[I-\Delta^{-} A(t)\right]^{-1}\right\|_{L(X)}<\infty: t \in(a, b]\right\}, \\
\|x(t)\|_{X} \leq c_{A}\left(\|\widetilde{x}\|_{X}+\|f(a)\|_{X}+\|f\|_{\infty}\right) \exp \left(c_{A} \operatorname{var}_{a}^{t} A\right) \quad \text { for } t \in[a, b] .
\end{gathered}
$$

Proof. The equalities (2.2) and (2.3) follow from [34, Proposition 2.3].

For any division $D=\left\{\alpha_{0}, \alpha_{1}, \ldots, \alpha_{\nu(D)}\right\}$ of $[a, b]$, by Remark 1.5, we have

$$
\begin{aligned}
& \sum_{j=1}^{\nu(D)}\left\|x\left(\alpha_{j}\right)-f\left(\alpha_{j}\right)-x\left(\alpha_{j-1}\right)+f\left(\alpha_{j-1}\right)\right\|_{X} \\
& \quad=\sum_{j=1}^{\nu(D)}\left\|\int_{\alpha_{j-1}}^{\alpha_{j}} \mathrm{~d}[A(s)] x(s)\right\|_{X} \leq \sum_{j=1}^{\nu(D)}\left[\left(\operatorname{var}_{\alpha_{j-1}}^{\alpha_{j}} A\right)\|x\|_{\infty}\right]=\left(\operatorname{var}_{a}^{b} A\right)\|x\|_{\infty} .
\end{aligned}
$$

Then, taking the supremum over all divisions of $[a, b]$, we obtain (2.4).

When $t \in[a, b]$ is such that $\left\|\Delta^{-} A(t)\right\|_{L(X)}<\frac{1}{2}$, it is known that

$$
\left\|\left[I-\Delta^{-} A(t)\right]^{-1}\right\|_{L(X)} \leq \frac{1}{1-\left\|\Delta^{-} A(t)\right\|_{L(X)}}<2
$$

(cf. e.g. [40, Theorem IV.1.4]). On the other hand, since the set

$$
\left\{t \in[a, b] ;\left\|\Delta^{-} A(t)\right\|_{L(X)} \geq \frac{1}{2}\right\}
$$

is finite (cf. [22, Corollary I.3.2.a]), we have clearly $c_{A}<\infty$.

Let $B:[a, b] \rightarrow L(X)$ be defined by $B(a)=A(a)$ and $B(t)=A(t-)$ for $t \in(a, b]$. By [34, Corollary 2.6] and [34, Proposition 2.7], we get

$$
A-B \in B V([a, b], L(X)), \quad \operatorname{var}_{a}^{b} B \leq \operatorname{var}_{a}^{b} A
$$

and

$$
A(t)-B(t)=\Delta^{-} A(t), \quad \int_{a}^{t} \mathrm{~d}[A-B] x=\Delta^{-} A(t) x(t) \quad \text { for } t \in(a, b]
$$


Hence,

$$
\left[I-\Delta^{-} A(t)\right] x(t)=\widetilde{x}+\int_{a}^{t} \mathrm{~d}[B(s)] x(s)+f(t)-f(a) \quad \text { for } t \in(a, b],
$$

or equivalently,

$$
x(t)=\left[I-\Delta^{-} A(t)\right]^{-1}\left(\widetilde{x}+\int_{a}^{t} \mathrm{~d}[B(s)] x(s)+f(t)-f(a)\right) .
$$

Taking into account (2.5) and Remark 1.5, we obtain

$$
\|x(t)\|_{X} \leq K_{1}+K_{2} \int_{a}^{t} \mathrm{~d}[h(s)]\|x(s)\|_{X} \quad \text { for } t \in[a, b],
$$

where $K_{1}=c_{A}\left(\|\widetilde{x}\|_{X}+2\|f\|_{\infty}\right), K_{2}=c_{A}$ and $h(s)=\operatorname{var}_{a}^{s} B, s \in[a, b]$. Noticing that $h$ is nondecreasing and left-continuous on $(a, b]$, the relation (2.6) can be derived by applying the generalized Gronwall inequality (see e.g. [32, Corollary 1.43] or [38, Lemma I.4.30].)

Lemma 2.3. Let $A, A_{n} \in B V([a, b], L(X)), n \in \mathbb{N}$, be such that (E) and

$$
\lim _{n \rightarrow \infty}\left\|A_{n}-A\right\|_{\infty}=0
$$

are satisfied. Then

$$
\left[I-\Delta^{-} A_{n}(t)\right]^{-1} \in L(X), \quad t \in(a, b]
$$

for $n \in \mathbb{N}$ sufficiently large. Moreover, there is $\mu^{*} \in(0, \infty)$ such that

$$
c_{A_{n}}:=\sup \left\{\left\|\left[I-\Delta^{-} A_{n}(t)\right]^{-1}\right\|_{L(X)}: t \in(a, b]\right\} \leq \mu^{*}
$$

for $n \in \mathbb{N}$ sufficiently large.

Proof. Notice that, by (2.7), $\lim _{n \rightarrow \infty}\left\|\Delta^{-} A_{n}-\Delta^{-} A\right\|_{\infty}=0$. Hence, there is $n_{0} \in \mathbb{N}$ such that

$$
\left\|\Delta^{-} A_{n}(t)-\Delta^{-} A(t)\right\|_{L(X)}<\frac{1}{4} \min \left\{1, \frac{1}{c_{A}}\right\}, \quad \text { for } t \in(a, b] \text { and } n \geq n_{0},
$$

where $c_{A}$ is given by (2.5).

Consider the finite set $D:=\left\{t \in(a, b] ;\left\|\Delta^{-} A(t)\right\|_{L(X)} \geq \frac{1}{4}\right\}$ (see [22, Corollary I.3.2.a]). Then, for $t \in[a, b] \backslash D$ and $n \geq n_{0}$, we have

$$
\left\|\Delta^{-} A_{n}(t)\right\|_{L(X)} \leq\left\|\Delta^{-} A_{n}(t)-\Delta^{-} A(t)\right\|_{L(X)}+\left\|\Delta^{-} A(t)\right\|_{L(X)}<\frac{1}{2},
$$


which, by [40, Theorem IV.1.4], implies that $I-\Delta^{-} A_{n}(t)$ is invertible and

$$
\left\|\left[I-\Delta^{-} A_{n}(t)\right]^{-1}\right\|_{L(X)}<2, \quad \text { for } t \in[a, b] \backslash D \text { and } n \geq n_{0}
$$

On the other hand, given $t \in D$ and $n \geq n_{0}$, write

$$
I-\Delta^{-} A_{n}(t)=\left(I-\Delta^{-} A(t)\right)\left(I-T_{n}(t)\right)
$$

where $T_{n}(t)=\left[I-\Delta^{-} A(t)\right]^{-1}\left(\Delta^{-} A_{n}(t)-\Delta^{-} A(t)\right)$. Thus, in order to prove that $I-\Delta^{-} A_{n}(t)$ is invertible, it is sufficient to show that $\left[I-T_{n}(t)\right]^{-1}$ exists.

Due to (2.5) and (2.10), we have

$$
\left\|T_{n}(t)\right\|_{L(X)} \leq\left\|\left[I-\Delta^{-} A(t)\right]^{-1}\right\|_{L(X)}\left\|\Delta^{-} A_{n}(t)-\Delta^{-} A(t)\right\|_{L(X)}<\frac{1}{4} .
$$

Thus, by [40, Lemma 4.1-C], $I-T_{n}(t)$ is invertible with $\left\|\left[I-T_{n}(t)\right]^{-1}\right\|_{L(X)}<2$ which, together with (2.11), leads to

$$
\left\|\left[I-\Delta^{-} A_{n}(t)\right]^{-1}\right\|_{L(X)}<2 c_{A} \quad \text { for } t \in D \text { and } n \geq n_{0} .
$$

In summary, (2.8) and (2.9) hold for $n \geq n_{0}$ and $\mu^{*}:=2 \max \left\{1, c_{A}\right\}$.

\subsection{Continuous dependence on parameters: variations bounded by a weight}

In this section, we deal with the homogeneous linear integral equation

$$
x(t)=\widetilde{x}+\int_{a}^{t} \mathrm{~d}[A] x, \quad t \in[a, b]
$$

where $\widetilde{x} \in X$ and $A \in B V([a, b], L(X))$.

The main result of this section is provided by the next theorem. It generalizes, in some sense, Theorem 1 from [30] due to Z. Opial, which is stated for the case when $X$ is the Euclidean space and the operator-valued functions $A, A_{n}, n \in \mathbb{N}$, are absolutely continuous on $[a, b]$. 
Theorem 2.4. Let $A, A_{n} \in B V([a, b], L(X))$ and $\widetilde{x}, \widetilde{x}_{n} \in X$ for all $n \in \mathbb{N}$. Let $A$ satisfy $(\mathrm{E})$ and $x$ be the solution of $(\mathrm{H})$ on $[a, b]$. Further, assume

$$
\lim _{n \rightarrow \infty}\left\|A_{n}-A\right\|_{\infty}\left(1+\operatorname{var}_{a}^{b} A_{n}\right)=0
$$

and

$$
\lim _{n \rightarrow \infty}\left\|\widetilde{x}_{n}-\widetilde{x}\right\|_{X}=0
$$

Then, for $n \in \mathbb{N}$ sufficiently large, the equation

$$
x_{n}(t)=\widetilde{x}_{n}+\int_{a}^{t} \mathrm{~d}\left[A_{n}\right] x_{n}, \quad t \in[a, b]
$$

has a unique solution $x_{n}$ on $[a, b]$ and

$$
\lim _{k \rightarrow \infty}\left\|x_{n}-x\right\|_{\infty}=0
$$

Proof. At first, notice that, since

$$
\left\|A_{n}-A\right\|_{\infty} \leq\left\|A_{n}-A\right\|_{\infty}\left(1+\operatorname{var}_{a}^{b} A_{n}\right) \text { for all } n \in \mathbb{N}
$$

(2.12) implies (2.7). Therefore, by Lemma 2.3, there is $n_{0} \in \mathbb{N}$ such that (2.8) holds for each $n \geq n_{0}$, which ensures the existence of a solution of (2.14) (cf. Proposition 2.1).

Assume $n \geq n_{0}$ and let $x$ and $x_{n}$ be the solutions on $[a, b]$ of $(\mathrm{H})$ and (2.14) respectively. Consider the equation

$$
x_{n}(t)-x(t)=\widetilde{x}_{n}-\widetilde{x}+\int_{a}^{t} \mathrm{~d}[A]\left(x_{n}-x\right)+h_{n}(t)-h_{n}(a) \text { for } t \in[a, b],
$$

where

$$
h_{n}(t)=\int_{a}^{t} \mathrm{~d}\left[A_{n}-A\right] x_{n} \quad \text { for } t \in[a, b] .
$$

By Lemma 2.2, we have

$$
\left\|x_{n}-x\right\|_{\infty} \leq c_{A}\left(\left\|\widetilde{x}_{n}-\widetilde{x}\right\|_{X}+\left\|h_{n}\right\|_{\infty}\right) \exp \left(c_{A} \operatorname{var}_{a}^{b} A\right)
$$

In view of this inequality and regarding the assumption (2.13), to complete the proof it is enough to show that $\lim _{n \rightarrow \infty}\left\|h_{n}\right\|_{\infty}=0$. 
Applying the Integration by Parts theorem (cf. Corollary 1.12) to the right-hand side of (2.16) and using the Substitution formula (cf. Theorem 1.14), we get

$$
\begin{aligned}
h_{n}(t) & =\left[A_{n}(t)-A(t)\right] x_{n}(t)-\left[A_{n}(a)-A(a)\right] \widetilde{x}_{n}-\int_{a}^{t}\left(A_{n}-A\right) \mathrm{d}\left[x_{n}\right]-\Delta_{a}^{t}\left(A_{n}-A, x_{n}\right) \\
& =\left[A_{n}(t)-A(t)\right] x_{n}(t)-\left[A_{n}(a)-A(a)\right] \widetilde{x}_{n}-\int_{a}^{t}\left(A_{n}-A\right) \mathrm{d}\left[A_{n}\right] x_{n}-\Delta_{a}^{t}\left(A_{n}-A, x_{n}\right)
\end{aligned}
$$

for $t \in[a, b]$, where

$$
\Delta_{a}^{t}\left(A_{n}-A, x_{n}\right)=\sum_{a \leq s<t}\left[\Delta^{+}\left(A_{n}(s)-A(s)\right) \Delta^{+} x_{n}(s)\right]-\sum_{a<s \leq t}\left[\Delta^{-}\left(A_{n}(s)-A(s)\right) \Delta^{-} x_{n}(s)\right]
$$

Inserting the relations (2.2) and (2.3) (with $f \equiv 0$ ) in the equality above, we get

$$
\begin{aligned}
& \Delta_{a}^{t}\left(A_{n}-A, x_{n}\right) \\
& =\sum_{a \leq s<t}\left[\Delta^{+}\left(A_{n}(s)-A(s)\right) \Delta^{+} A_{n}(s) x_{n}(s)\right]-\sum_{a<s \leq t}\left[\Delta^{-}\left(A_{n}(s)-A(s)\right) \Delta^{-} A_{n}(s) x_{n}(s)\right],
\end{aligned}
$$

from where, by Lemma 1.2, it follows that

$$
\left\|\Delta_{a}^{t}\left(A_{n}-A, x_{n}\right)\right\|_{X} \leq 2\left\|A_{n}-A\right\|_{\infty}\left(\operatorname{var}_{a}^{t} A_{n}\right)\left\|x_{n}\right\|_{\infty} \text { for } t \in[a, b] .
$$

Moreover, using arguments analogous to those found in the proof of [33, Proposition 10], one can prove that the estimate

$$
\left\|\int_{a}^{t}\left(A_{n}-A\right) \mathrm{d}\left[A_{n}\right] x_{n}\right\|_{X} \leq\left\|A_{n}-A\right\|_{\infty} \operatorname{var}_{a}^{b} A_{n}\left\|x_{n}\right\|_{\infty}
$$

holds. Therefore, $\left\|h_{n}\right\|_{\infty} \leq \alpha_{n}\left\|x_{n}\right\|_{\infty}$, where

$$
\alpha_{n}:=\left\|A_{n}-A\right\|_{\infty}\left(2+3 \operatorname{var}_{a}^{b} A_{n}\right) \quad \text { with } \lim _{n \rightarrow \infty} \alpha_{n}=0
$$

Note that, $\left\|x_{n}\right\|_{\infty}, n \geq n_{0}$, defines a bounded sequence in $\mathbb{R}$. Indeed, by (2.17)

$$
\left\|x_{n}\right\|_{\infty} \leq\left\|x_{n}-x\right\|_{\infty}+\|x\|_{\infty} \leq c_{A}\left(\left\|\widetilde{x}_{n}-\widetilde{x}\right\|_{X}+\alpha_{n}\left\|x_{n}\right\|_{\infty}\right) \exp \left(c_{A} \operatorname{var}_{a}^{b} A\right)+\|x\|_{\infty},
$$

or equivalently,

$$
\left(1-c_{A} \alpha_{n} \exp \left(c_{A} \operatorname{var}_{a}^{b} A\right)\right)\left\|x_{n}\right\|_{\infty} \leq c_{A}\left\|\widetilde{x}_{n}-\widetilde{x}\right\|_{X} \exp \left(c_{A} \operatorname{var}_{a}^{b} A\right)+\|x\|_{\infty}
$$


By (2.13) and (2.18), there is $n_{1} \geq n_{0}$ such that

$$
\left\|\widetilde{x}_{n}-\widetilde{x}\right\|_{X}<1 \quad \text { and } \quad c_{A} \alpha_{n} \exp \left(c_{A} \operatorname{var}_{a}^{b} A\right)<\frac{1}{2} \quad \text { for } n \geq n_{1} .
$$

Thus, $\left\|x_{n}\right\|_{\infty}<2\left(c_{A} \exp \left(c_{A} \operatorname{var}_{a}^{b} A\right)+\|x\|_{\infty}\right)$ for $n \geq n_{1}$ which, together with (2.18), proves that $\lim _{n \rightarrow \infty}\left\|h_{n}\right\|_{\infty}=0$ and, consequently, the theorem holds.

In [30], Z. Opial notes that his main theorem can be extended to nonhomogeneous equations treating them as special homogeneous problems. Using such procedure, well-known in classical theory of ordinary differential equations, it is possible to see that Theorem 2.4 is also true for nonhomogeneous problems when $X$ is the Euclidean space $\mathbb{R}^{m}$, for some $m \in \mathbb{N}$.

To make it clear, let us recall that given $A:[a, b] \rightarrow L\left(\mathbb{R}^{m}\right)$ and $f:[a, b] \rightarrow \mathbb{R}^{m}$, we can define a $(m+1) \times(m+1)$-matrix valued function $B:[a, b] \rightarrow L\left(\mathbb{R}^{m+1}\right)$ by

$$
B(t)=\left(\begin{array}{cc}
A(t) & f(t) \\
0 & 0
\end{array}\right) \quad \text { for } t \in[a, b]
$$

It is easy to check that if $x$ is a solution to $(\mathrm{NH})$ and $y(t)=\left(\begin{array}{c}x(t) \\ 1\end{array}\right)$, then $y$ is a solution to the homogeneous equation

$$
y(t)=\widetilde{y}+\int_{a}^{t} \mathrm{~d}[B] y, \quad \text { with } \quad \widetilde{y}=\left(\begin{array}{l}
\widetilde{x} \\
1
\end{array}\right)
$$

Conversely, if $y$ is a solution of (2.19) and $x$ is formed by its first $m$ components then $x$ is a solution to $(\mathrm{NH})$, where $\widetilde{x} \in \mathbb{R}^{m}$ is formed by the first $m$ components of $\widetilde{y}$.

Similarly, we define

$$
B_{n}(t)=\left(\begin{array}{cc}
A_{n}(t) & f_{n}(t) \\
0 & 0
\end{array}\right) \quad \text { for } t \in[a, b] \quad \text { and } n \in \mathbb{N} .
$$

where $A_{n}:[a, b] \rightarrow L\left(\mathbb{R}^{m}\right)$ and $f_{n}:[a, b] \rightarrow \mathbb{R}^{m}, n \in \mathbb{N}$. And again, for each $n \in \mathbb{N}$, we have the correspondence between the solutions of

$$
y_{n}(t)=\widetilde{y}_{n}+\int_{a}^{t} \mathrm{~d}\left[B_{n}\right] y_{n}, t \in[a, b], \quad \text { with } \quad \widetilde{y}_{n}=\left(\begin{array}{c}
\widetilde{x}_{n} \\
1
\end{array}\right),
$$


and

$$
x_{n}(t)=\widetilde{x}_{n}+\int_{a}^{t} \mathrm{~d}\left[A_{n}\right] x_{n}+f_{n}(t)-f_{n}(a), t \in[a, b] .
$$

In view of these remarks, the next assertion is a direct consequence of Theorem 2.4.

Corollary 2.5. Let $m \in \mathbb{N}, A, A_{n} \in B V\left([a, b], L\left(\mathbb{R}^{m}\right)\right), f, f_{n} \in B V\left([a, b], \mathbb{R}^{m}\right)$, and $\widetilde{x}, \widetilde{x}_{n} \in \mathbb{R}^{m}$ for $n \in \mathbb{N}$. Assume (E), (2.13) and

$$
\begin{aligned}
& \lim _{n \rightarrow \infty}\left(\left\|A_{n}-A\right\|_{\infty}\left(1+\operatorname{var}_{a}^{b} A_{n}+\operatorname{var}_{a}^{b} f_{n}\right)\right)=0 \\
& \lim _{n \rightarrow \infty}\left(\left\|f_{n}-f\right\|_{\infty}\left(1+\operatorname{var}_{a}^{b} A_{n}+\operatorname{var}_{a}^{b} f_{n}\right)\right)=0
\end{aligned}
$$

Then equation $(\mathrm{NH})$ has a unique solution $x$ on $[a, b]$ and, for each $n \in \mathbb{N}$ large enough there is a unique solution $x_{n}$ on $[a, b]$ to the equation (2.20) and (2.15) is true.

Remark 2.6. Note that in Corollary 2.5, we require the functions $f, f_{n}, n \in \mathbb{N}$, being of bounded variation and not only regulated. Such condition is needed to ensure that $B$ and $B_{n}, n \in \mathbb{N}$, define functions of bounded variation, once we have

$$
\operatorname{var}_{a}^{b} B \leq \operatorname{var}_{a}^{b} A+\operatorname{var}_{a}^{b} f \quad \text { and } \quad \operatorname{var}_{a}^{b} B_{n} \leq \operatorname{var}_{a}^{b} A_{n}+\operatorname{var}_{a}^{b} f_{n}, n \in \mathbb{N}
$$

\subsection{Continuous dependence on parameters: uniformly bounded variation}

The main result of this section concerns the continuous dependence on parameters for solutions of nonhomogeneous linear integral equations of type (NH). It recalls the result by M. Ashordia for linear generalized ODEs in the framework of $\mathbb{R}^{m}$-valued functions, $m \in \mathbb{N}$ (see [3, Theorem 1]). However, unlike [3], no variation-of-constants formula is used. Thus, we do not need to require the additional condition $\left[I+\Delta^{+} A(t)\right]^{-1} \in L(X)$ for $t \in[a, b]$; condition (E) remains enough.

The proof presented below follows the ideas provided by Z. Halas and M. Tvrdý in [18], where an extention of Ashordia's result to the complex case is given. 
Theorem 2.7. Let $A, A_{n} \in B V([a, b], L(X)), f, f_{n} \in G([a, b], X)$ and $\widetilde{x}, \widetilde{x}_{n} \in X$ for all $n \in \mathbb{N}$. Let $A$ satisfy (E) and $x$ be the solution of (NH). Assume (2.7), (2.13) and also

$$
\begin{gathered}
\alpha^{*}:=\sup _{n \in \mathbb{N}}\left(\operatorname{var}_{a}^{b} A_{n}\right)<\infty, \\
\lim _{n \rightarrow \infty}\left\|f_{n}-f\right\|_{\infty}=0 .
\end{gathered}
$$

Then, for $n \in \mathbb{N}$ sufficiently large, there is a unique solution $x_{n}$ on $[a, b]$ of equation

$$
x_{n}(t)=\widetilde{x}_{n}+\int_{a}^{t} \mathrm{~d}\left[A_{n}\right] x_{n}+f_{n}(t)-f_{n}(a), \quad t \in[a, b]
$$

and (2.15) holds.

Proof. Note that, by Lemma 2.3, there exists $n_{0} \in \mathbb{N}$ such that (2.8) is true for $n \geq n_{0}$. Hence, for each $n \geq n_{0}$, equation (2.25) has a unique solution $x_{n}$ on $[a, b]$.

Set $w_{n}=\left(x_{n}-f_{n}\right)-(x-f)$ and consider the equation

$$
w_{n}(t)=\widetilde{w}_{n}+\int_{a}^{t} \mathrm{~d}\left[A_{n}\right] w_{n}+h_{n}(t)-h_{n}(a) \text { for } t \in[a, b],
$$

where $\widetilde{w}_{n}=\left(\widetilde{x}_{n}-f_{n}(a)\right)-(\widetilde{x}-f(a))$ and

$$
h_{n}(t)=\left(\int_{a}^{t} \mathrm{~d}\left[A_{n}\right] f_{n}-\int_{a}^{t} \mathrm{~d}[A] f\right)+\int_{a}^{t} \mathrm{~d}\left[A_{n}-A\right](x-f) .
$$

Let $\mu^{*}>0$ be as in (2.9). By Lemma 2.2 and by (2.23), we have

$$
\left\|w_{n}(t)\right\|_{X} \leq c_{A_{n}}\left(\left\|\widetilde{w}_{n}\right\|_{X}+\left\|h_{n}\right\|_{\infty}\right) \exp \left(c_{A_{n}} \operatorname{var}_{a}^{b} A_{n}\right)
$$

that is,

$$
\left\|w_{n}(t)\right\|_{X} \leq \mu^{*}\left(\left\|\widetilde{w}_{n}\right\|_{X}+\left\|h_{n}\right\|_{\infty}\right) \exp \left(\mu^{*} \alpha^{*}\right) \text { for } t \in[a, b]
$$

Note that (2.13) and (2.24) imply that $\lim _{n \rightarrow \infty}\left\|\widetilde{w}_{n}\right\|_{X}=0$. Also, since $x-f$ is a function of bounded variation on $[a, b]$ (cf. Lemma 2.2), using the relation (1.7), we get

$$
\left\|h_{n}\right\|_{\infty} \leq\left(\sup _{t \in[a, b]}\left\|\int_{a}^{t} \mathrm{~d}\left[A_{n}\right] f_{n}-\int_{a}^{t} \mathrm{~d}[A] f\right\|_{X}\right)+2\left\|A_{n}-A\right\|_{\infty}\|x-f\|_{B V} .
$$

Then, in view of Theorem 1.6 and (2.7), $\lim _{n \rightarrow \infty}\left\|h_{n}\right\|_{\infty}=0$. 
Finally, by the inequality (2.26), we deduce that

$$
\lim _{n \rightarrow \infty}\left\|\left(x_{n}-f_{n}\right)-(x-f)\right\|_{\infty}=\lim _{n \rightarrow \infty}\left\|w_{n}\right\|_{\infty}=0
$$

which, together with (2.24), leads to (2.15).

We will close this section with a comparison between Theorem 2.7 and two similar available results: Proposition 8.3 in [1] (see also [32, Theorem 8.2] for the finite dimensional case) and Theorem 8.8 from [32]. To this aim, let us recall the class of functions introduced by J. Kurzweil in his papers [24] and [25] (see also [32, Definition 3.8]).

Let $r>0$ be given and let us set $\Omega=B_{r} \times[a, b]$, where $B_{r}:=\left\{x \in X:\|x\|_{X} \leq r\right\}$. In the sequell, we assume $h:[a, b] \rightarrow \mathbb{R}$ is a nondecreasing function and $\omega:[0, \infty) \rightarrow \mathbb{R}$ is a continuous increasing function with $\omega(0)=0$.

Definition 2.8. A function $F: \Omega \rightarrow X$ belongs to the class $\mathcal{F}(\Omega, h, \omega)$ if

$$
\left\|F\left(x, t_{2}\right)-F\left(x, t_{1}\right)\right\|_{X} \leq\left|h\left(t_{2}\right)-h\left(t_{1}\right)\right|
$$

for all $\left(x, t_{2}\right),\left(x, t_{1}\right) \in \Omega$ and

$$
\left\|F\left(x, t_{2}\right)-F\left(x, t_{1}\right)-F\left(y, t_{2}\right)+F\left(y, t_{1}\right)\right\|_{X} \leq \omega\left(\|x-y\|_{X}\right)\left|h\left(t_{2}\right)-h\left(t_{1}\right)\right|
$$

for all $\left(x, t_{2}\right),\left(x, t_{1}\right),\left(y, t_{2}\right),\left(y, t_{1}\right) \in \Omega$.

In particular, when $F(x, t)=A(t) x+f(t)$, with $A:[a, b] \rightarrow L(X)$ and $f:[a, b] \rightarrow X$, we get

$$
\begin{aligned}
& \left\|\left[A\left(t_{2}\right)-A\left(t_{1}\right)\right] x+f\left(t_{2}\right)-f\left(t_{1}\right)\right\|_{X} \leq\left|h\left(t_{2}\right)-h\left(t_{1}\right)\right|, \\
& \left\|\left[A\left(t_{2}\right)-A\left(t_{1}\right)\right](y-x)\right\|_{X} \leq \omega\left(\|y-x\|_{X}\right)\left|h\left(t_{2}\right)-h\left(t_{1}\right)\right|,
\end{aligned}
$$

for $t_{1}, t_{2} \in[a, b]$ and $x, y \in B_{r}$.

With (2.27) in mind, Theorem 8.2 from [32] can be written as follows.

Theorem 2.9. Let $A, A_{n}:[a, b] \rightarrow L(X), f, f_{n}:[a, b] \rightarrow X$ and $\widetilde{x}_{n}, \widetilde{x} \in X$ for all $n \in \mathbb{N}$ and let $r>1$. Assume (2.13), (2.27) and

$$
\begin{aligned}
& \left\|\left[A_{n}\left(t_{2}\right)-A_{n}\left(t_{1}\right)\right] x+f_{n}\left(t_{2}\right)-f_{n}\left(t_{1}\right)\right\|_{X} \leq\left|h\left(t_{2}\right)-h\left(t_{1}\right)\right|, \\
& \left\|\left[A_{n}\left(t_{2}\right)-A_{n}\left(t_{1}\right)\right](y-x)\right\|_{X} \leq \omega\left(\|y-x\|_{X}\right)\left|h\left(t_{2}\right)-h\left(t_{1}\right)\right|,
\end{aligned}
$$


for $t_{1}, t_{2} \in[a, b], x, y \in B_{r}$ and $n \in \mathbb{N}$. Assume further that

$$
\lim _{n \rightarrow \infty}\left\|A_{n}(t)-A(t)\right\|_{L(X)}=0 \quad \text { and } \quad \lim _{n \rightarrow \infty}\left\|f_{n}(t)-f(t)\right\|_{X}=0 \quad \text { for } t \in[a, b] .
$$

Let $x_{n}$ be solutions of (2.25) for all $n \in \mathbb{N}$ and let $x:[a, b] \rightarrow X$ be such that

$$
\lim _{n \rightarrow \infty}\left\|x_{n}(t)-x(t)\right\|_{X}=0 \quad \text { and } \quad x(t) \in B_{r} \quad \text { for } t \in[a, b] .
$$

Then $x \in B V([a, b], X)$ and it is a solution of $(\mathrm{NH})$ on $[a, b]$.

Similarly, when restricted to the linear case, Theorem 8.8 from [32] reduces to the next result.

Theorem 2.10. Let $X=\mathbb{R}^{m}$ for some $m \in \mathbb{N}$. Let $A, A_{n}:[a, b] \rightarrow L(X), f, f_{n}:[a, b] \rightarrow X$, and $\widetilde{x}_{n}, \widetilde{x} \in X$ for all $n \in \mathbb{N}$. Given $r>1$, suppose that (E), (2.13) and (2.29) are satisfied. Further, assume (2.27) holds with h continuous and

$$
\begin{aligned}
& \left\|\left[A_{n}\left(t_{2}\right)-A_{n}\left(t_{1}\right)\right] x+f_{n}\left(t_{2}\right)-f_{n}\left(t_{1}\right)\right\|_{X} \leq\left|h_{n}\left(t_{2}\right)-h_{n}\left(t_{1}\right)\right|, \\
& \left\|\left[A_{n}\left(t_{2}\right)-A_{n}\left(t_{1}\right)\right](y-x)\right\|_{X} \leq \omega\left(\|y-x\|_{X}\right)\left|h_{n}\left(t_{2}\right)-h_{n}\left(t_{1}\right)\right|,
\end{aligned}
$$

for $t_{1}, t_{2} \in[a, b], x, y \in B_{r}$ and $n \in \mathbb{N}$, where $h_{n}:[a, b] \rightarrow \mathbb{R}, n \in \mathbb{N}$, are nondecreasing, left continuous functions such that

$$
\limsup _{n \rightarrow \infty}\left[h_{n}\left(t_{2}\right)-h_{n}\left(t_{1}\right)\right] \leq h\left(t_{2}\right)-h\left(t_{1}\right) \quad \text { whenever } a \leq t_{1} \leq t_{2} \leq b
$$

If $x$ is the solution of $(\mathrm{NH})$, then for $n \in \mathbb{N}$ sufficiently large, equation (2.25) has a unique solution $x_{n}$ on $[a, b]$ and (2.15) holds.

The following two propositions establish a connection between the assumptions of Theorem 2.7 and the previous continuous dependence results, namely Theorems 2.9 and 2.10. The proofs we present here were inspired in a similar type of comparison due to M. Tvrdý [43], in case $X$ has finite dimension.

Proposition 2.11. Let $A, A_{n}:[a, b] \rightarrow L(X)$ and $f, f_{n}:[a, b] \rightarrow X$ for $n \in \mathbb{N}$. Assume (2.27) to (2.29) hold. Then $A_{n}, A \in B V([a, b], L(X)), f_{n}, f \in B V([a, b], X)$ for all $n \in \mathbb{N}$ and the relations (2.7), (2.23) and (2.24) are satisfied. 
Proof. First of all, note that, taking $x=0$ in (2.27) and (2.28), we get

$$
\begin{aligned}
& \left\|A\left(t_{2}\right)-A\left(t_{1}\right)\right\|_{L(X)} \leq \omega(1)\left|h\left(t_{2}\right)-h\left(t_{1}\right)\right|, \\
& \left\|A_{n}\left(t_{2}\right)-A_{n}\left(t_{1}\right)\right\|_{L(X)} \leq \omega(1)\left|h\left(t_{2}\right)-h\left(t_{1}\right)\right|
\end{aligned} \quad \text { for } t_{1}, t_{2} \in[a, b], n \in \mathbb{N}
$$

and

$$
\begin{aligned}
& \left\|f\left(t_{2}\right)-f\left(t_{1}\right)\right\|_{X} \leq\left|h\left(t_{2}\right)-h\left(t_{1}\right)\right|, \\
& \left\|f_{n}\left(t_{2}\right)-f_{n}\left(t_{1}\right)\right\|_{X} \leq\left|h\left(t_{2}\right)-h\left(t_{1}\right)\right|
\end{aligned} \quad \text { for } t_{1}, t_{2} \in[a, b], n \in \mathbb{N} .
$$

In view of these inequalities, it is clear that $A, A_{n}, f, f_{n}, n \in \mathbb{N}$, are functions of bounded variation on $[a, b]$. Moreover,

$$
\operatorname{var}_{a}^{b} A_{n} \leq \omega(1)(h(b)-h(a)), \quad \text { for all } n \in \mathbb{N}
$$

and (2.23) follows.

Now, let us prove the relation (2.7), that is, $\lim _{n \rightarrow \infty}\left\|A_{n}-A\right\|_{\infty}=0$.

Step 1. Notice that (2.32) and (2.29) imply that

$$
\begin{aligned}
& \|A(t-)-A(s)\|_{L(X)} \leq \omega(1)|h(t-)-h(s)|, \\
& \left\|A_{n}(t-)-A_{n}(s)\right\|_{L(X)} \leq \omega(1)|h(t-)-h(s)|
\end{aligned} \quad \text { for } t \in(a, b], s \in[a, b], n \in \mathbb{N}
$$

and

$$
\begin{aligned}
& \|A(t+)-A(s)\|_{L(X)} \leq \omega(1)|h(t+)-h(s)|, \\
& \left\|A_{n}(t+)-A_{n}(s)\right\|_{L(X)} \leq \omega(1)|h(t+)-h(s)|
\end{aligned} \quad \text { for } t \in[a, b), s \in[a, b], n \in \mathbb{N} .
$$

Step 2. Given $\varepsilon>0$ and $t \in(a, b]$, let us choose $s_{0} \in(a, t)$ and $n_{0} \in \mathbb{N}$ such that

$$
\left|h(t-)-h\left(s_{0}\right)\right|<\frac{\varepsilon}{3 \omega(1)} \quad \text { and } \quad\left\|A_{n}\left(s_{0}\right)-A\left(s_{0}\right)\right\|_{L(X)}<\frac{\varepsilon}{3} \quad \text { for } n \geq n_{0} .
$$

Using these inequalities and (2.34), we get

$$
\begin{aligned}
& \left\|A_{n}(t-)-A(t-)\right\|_{L(X)} \\
& \quad \leq\left\|A_{n}(t-)-A_{n}\left(s_{0}\right)\right\|_{L(X)}+\left\|A_{n}\left(s_{0}\right)-A\left(s_{0}\right)\right\|_{L(X)}+\left\|A\left(s_{0}\right)-A(t-)\right\|_{L(X)} \\
& \quad<\omega(1)\left|h(t-)-h\left(s_{0}\right)\right|+\frac{\varepsilon}{3}+\omega(1)\left|h(t-)-h\left(s_{0}\right)\right|<\varepsilon
\end{aligned}
$$

which means

$$
\lim _{n \rightarrow \infty} A_{n}(t-)=A(t-) \quad \text { for } t \in(a, b] .
$$


Similarly, regarding (2.35), it follows that

$$
\lim _{n \rightarrow \infty} A_{n}(t+)=A(t+) \quad \text { for } t \in[a, b) .
$$

Step 3. Now, suppose that (2.7) is not valid. Then there is $\widetilde{\varepsilon}>0$ such that, for any $k \in \mathbb{N}$, there exist $m_{k} \geq k$ and $t_{k} \in[a, b]$ such that

$$
\left\|A_{m_{k}}\left(t_{k}\right)-A\left(t_{k}\right)\right\|_{L(X)} \geq \widetilde{\varepsilon}
$$

We can assume, without loss of generality, that $m_{k+1}>m_{k}$ for any $k \in \mathbb{N}$ and

$$
\lim _{k \rightarrow \infty} t_{k}=\bar{t} \in[a, b]
$$

Firstly, consider the case when $\bar{t} \in(a, b]$ and assume the interval $(a, \bar{t})$ has infinitely many elements of the sequence $\left\{t_{k}\right\}$, that is, $(a, \bar{t})$ contains a subsequence which we still denote by $\left\{t_{k}\right\}$. By (2.34),

$$
\begin{aligned}
& \left\|A(\bar{t}-)-A\left(t_{k}\right)\right\|_{L(X)} \leq \omega(1)\left[h(\bar{t}-)-h\left(t_{k}\right)\right], \\
& \left\|A_{m_{k}}(\bar{t}-)-A_{m_{k}}\left(t_{k}\right)\right\|_{L(X)} \leq \omega(1)\left[h(\bar{t}-)-h\left(t_{k}\right)\right]
\end{aligned} \quad \text { for } k \in \mathbb{N} .
$$

Thus, by (2.36) and since $\lim _{k \rightarrow \infty}\left(h(\bar{t}-)-h\left(t_{k}\right)\right)=0$, there is $k_{0} \in \mathbb{N}$ such that

$$
\begin{aligned}
& \left\|A_{m_{k_{0}}}(\bar{t}-)-A(\bar{t}-)\right\|_{L(X)}<\frac{\widetilde{\varepsilon}}{3}, \\
& \left\|A(\bar{t}-)-A\left(t_{k_{0}}\right)\right\|_{L(X)} \leq \omega(1)\left[h(\bar{t}-)-h\left(t_{k_{0}}\right)\right]<\frac{\widetilde{\varepsilon}}{3} \\
& \left\|A_{m_{k_{0}}}(\bar{t}-)-A_{m_{k_{0}}}\left(t_{k_{0}}\right)\right\|_{L(X)}<\frac{\widetilde{\varepsilon}}{3} .
\end{aligned}
$$

As a consequence, by (2.38), we finally get

$$
\begin{aligned}
\widetilde{\varepsilon} & \leq\left\|A_{m_{k_{0}}}\left(t_{k_{0}}\right)-A\left(t_{k_{0}}\right)\right\|_{L(X)} \\
& \leq\left\|A_{m_{k_{0}}}\left(t_{k_{0}}\right)-A_{m_{k_{0}}}(\bar{t}-)\right\|_{L(X)}+\left\|A_{m_{k_{0}}}(\bar{t}-)-A(\bar{t}-)\right\|_{L(X)}+\left\|A(\bar{t}-)-A\left(t_{k_{0}}\right)\right\|_{L(X)}<\widetilde{\varepsilon},
\end{aligned}
$$

which is a contradiction.

For the case when $\bar{t} \in[a, b)$ and the set of those $k \in \mathbb{N}$ for which $t_{k} \in(a, \bar{t})$ is only finite, there is a subsequence of $\left\{t_{k}\right\}$ contained in the interval $(\bar{t}, b)$ which we may denote again by $\left\{t_{k}\right\}$. 
Therefore, arguing similarly as before, there is $k_{0} \in \mathbb{N}$ such that

$$
\begin{aligned}
\widetilde{\varepsilon} & \leq\left\|A_{m_{k_{0}}}\left(t_{k_{0}}\right)-A\left(t_{k_{0}}\right)\right\|_{L(X)} \\
& \leq\left\|A_{m_{k_{0}}}\left(t_{k_{0}}\right)-A_{m_{k_{0}}}(\bar{t}+)\right\|_{L(X)}+\left\|A_{m_{k_{0}}}(\bar{t}+)-A(\bar{t}+)\right\|_{L(X)}+\left\|A(\bar{t}+)-A\left(t_{k_{0}}\right)\right\|_{L(X)}<\widetilde{\varepsilon},
\end{aligned}
$$

which is a contradiction. Thus (2.7) is satisfied.

To obtain (2.24), one can use the inequalities in (2.33) and follow the steps 1 to 3.

Proposition 2.12. Let $A, A_{n}:[a, b] \rightarrow L(X), f, f_{n}:[a, b] \rightarrow X$ for $n \in \mathbb{N}$ and let $h, h_{n}:[a, b] \rightarrow \mathbb{R}$, $n \in \mathbb{N}$, be as in Theorem 2.9. Furthermore, assume (2.27) and (2.29) to (2.31) hold. Then, for any $n \in \mathbb{N}$, we have $A_{n}, A \in B V([a, b], L(X)), f_{n}, f \in B V([a, b], X)$ and the relations (2.7), (2.23) and (2.24) are satisfied.

Proof. As in the proof of Proposition 2.11, taking $x=0$ in (2.27) and (2.30), we obtain

$$
\begin{aligned}
& \left\|A\left(t_{2}\right)-A\left(t_{1}\right)\right\|_{L(X)} \leq \omega(1)\left|h\left(t_{2}\right)-h\left(t_{1}\right)\right|, \\
& \left\|A_{n}\left(t_{2}\right)-A_{n}\left(t_{1}\right)\right\|_{L(X)} \leq \omega(1)\left|h_{n}\left(t_{2}\right)-h_{n}\left(t_{1}\right)\right|
\end{aligned} \quad \text { for } t_{1}, t_{2} \in[a, b], n \in \mathbb{N}
$$

and

$$
\begin{aligned}
& \left\|f\left(t_{2}\right)-f\left(t_{1}\right)\right\|_{X} \leq\left|h\left(t_{2}\right)-h\left(t_{1}\right)\right|, \\
& \left\|f_{n}\left(t_{2}\right)-f_{n}\left(t_{1}\right)\right\|_{X} \leq\left|h_{n}\left(t_{2}\right)-h_{n}\left(t_{1}\right)\right|
\end{aligned} \quad \text { for } t_{1}, t_{2} \in[a, b], n \in \mathbb{N} .
$$

By (2.31), there is $n_{0} \in \mathbb{N}$ such that $h_{n}(b)-h_{n}(a) \leq h(b)-h(a)+1$ for $n \geq n_{0}$. Hence, in view of (2.40), for any $n \in \mathbb{N}$, we have

$$
\operatorname{var}_{a}^{b} A_{n} \leq \max \left\{(h(b)-h(a)+1), \operatorname{var}_{a}^{b} A_{1}, \ldots, \operatorname{var}_{a}^{b} A_{n_{0}}\right\}<\infty,
$$

which proves (2.23).

Suppose that (2.7) does not hold. Then there is $\widetilde{\varepsilon}>0$ such that for any $k \in \mathbb{N}$, there exist $m_{k} \geq k$ and $t_{k} \in[a, b]$ such that $m_{k+1}>m_{k}$ for $k \in \mathbb{N}$ and the relations (2.38) and (2.39) are true.

At first, consider the case when $\bar{t} \in(a, b)$ and let an arbitrary $\varepsilon>0$ be given. Since $h$ is continuous, we may choose $\eta>0$ in such a way that

$$
h(\bar{t}+\eta)-h(\bar{t}-\eta)<\frac{\varepsilon}{\omega(1)} .
$$


Furthermore, by (2.29), there is $k_{1} \in \mathbb{N}$ such that

$$
\left\|A_{m_{k}}(\bar{t})-A(\bar{t})\right\|_{L(X)}<\varepsilon \quad \text { for all } k \geq k_{1}
$$

and, by (2.31), (2.40) and (2.42), there is $k_{2} \in \mathbb{N}, k_{2} \geq k_{1}$, such that

$$
\left\|A_{m_{k}}\left(\tau_{2}\right)-A_{m_{k}}\left(\tau_{1}\right)\right\|_{L(X)} \leq \omega(1)[h(\bar{t}+\eta)-h(\bar{t}-\eta)]+\varepsilon<2 \varepsilon
$$

whenever $\tau_{1}, \tau_{2} \in(\bar{t}-\eta, \bar{t}+\eta)$ and $k \geq k_{2}$. This, together with (2.29), implies immediately that

$$
\left\|A\left(\tau_{2}\right)-A\left(\tau_{1}\right)\right\|_{L(X)}=\lim _{k \rightarrow \infty}\left\|A_{m_{k}}\left(\tau_{2}\right)-A_{m_{k}}\left(\tau_{1}\right)\right\|_{L(X)} \leq 2 \varepsilon
$$

for any $\tau_{1}, \tau_{2} \in(\bar{t}-\eta, \bar{t}+\eta)$.

Finally, let $k_{3} \in \mathbb{N}$ be such that $k_{3} \geq k_{2}$ and

$$
\left|t_{k}-\bar{t}\right|<\eta \quad \text { for all } k \geq k_{3}
$$

Then, in view of inequalities (2.42) to (2.46), we have

$$
\begin{aligned}
& \left\|A_{m_{k}}\left(t_{k}\right)-A\left(t_{k}\right)\right\|_{L(X)} \\
& \quad \leq\left\|A_{m_{k}}\left(t_{k}\right)-A_{m_{k}}(\bar{t})\right\|_{L(X)}+\left\|A_{m_{k}}(\bar{t})-A(\bar{t})\right\|_{L(X)}+\left\|A(\bar{t})-A\left(t_{k}\right)\right\|_{L(X)} \leq 5 \varepsilon .
\end{aligned}
$$

Hence, choosing $\varepsilon<\frac{1}{5} \widetilde{\varepsilon}$ and making use of (2.38), we get $\widetilde{\varepsilon}>\left\|A_{m_{k}}\left(t_{k}\right)-A\left(t_{k}\right)\right\|_{L(X)} \geq \widetilde{\varepsilon}$ which is a contradiction. This proves that (2.7) is satisfied.

The adaptation of the proof for the cases $\bar{t}=a$ or $\bar{t}=b$ is obvious.

Finally, by (2.29) and (2.41), the inequalities (2.43) to (2.45) can be rewritten for the functions $f, f_{n}, n \in \mathbb{N}$. Consequently (2.24) also holds. 


\begin{tabular}{l|c|}
\hline CHAPTER \\
\cline { 2 - 2 } \\
\hline 3 \\
\hline
\end{tabular}

\section{Applications to dynamic equations on time scales}

The theory of time scales, whose initial studies are due to S. Hilger [20], has recently been receiving special attention. The reason is that this theory represents a unified treatment of both continuous and discrete analysis. This means that differential and difference equations can be regarded as particular cases of the so-called dynamic equations on time scales. Furthermore, the potential for applications of time scales calculus is remarkable. We can mention, for instance, population dynamics or the modeling of a simple electric circuit (cf. [2]).

In this chapter, based on the correspondence between dynamic equation and generalized differential equations established in [39], we apply some theorems from Chapter 2 to obtain new results on continuous dependence on parameter for dynamic equations on time scales. 


\subsection{Basic results and definitions}

Throughout this chapter we restrict ourselves to problems involving $\mathbb{R}^{m}$-valued functions, for some fixed $m \in \mathbb{N}$. We remind the reader that any norm in $\mathbb{R}^{m}$ is denoted by single bars, $|\cdot|$, while the operator norm in $L\left(\mathbb{R}^{m}\right)$ is represented by $\|\cdot\|$.

A time scale is a nonempty closed subset of $\mathbb{R}$ which we denote by $\mathbb{T}$. The real numbers, the integers, the natural numbers are well-known examples of time scales, as are the Cantor set and the groups $h \mathbb{Z}=\{h q: q \in \mathbb{Z}\}$ for $h>0$.

Given $a, b \in \mathbb{T},[a, b]_{\mathbb{T}}$ stands for the compact interval in $\mathbb{T}$, that is, $[a, b]_{\mathbb{T}}=[a, b] \cap \mathbb{T}$.

As usual, for each $t \in \mathbb{T}$, we define the jump operators by

$$
\rho(t):=\sup \{s \in \mathbb{T}: s<t\} \quad \text { and } \quad \sigma(t):=\inf \{s \in \mathbb{T}: s>t\}
$$

If $\sigma(t)=t$, we say that $\mathrm{t}$ is right-dense, while if $\rho(t)=t$ then $t$ is called left-dense.

A function $f:[a, b]_{\mathbb{T}} \rightarrow \mathbb{R}^{m}$ is rd-continuous on $[a, b]_{\mathbb{T}}$, if $f$ is continuous at every right-dense point of $[a, b]_{\mathbb{T}}$ and the left-sided limit, $f(t-)$, exists for every left-dense point $t \in[a, b]_{\mathbb{T}}$. Clearly, every rd-continuous function is also regulated.

In the following lines we recall some terminology and notations from time scales calculus which we will be needed later. For a more comprehensive study of this topic, see [4] and [5], for instance.

Define $\mathbb{T}^{\kappa}=\mathbb{T}$, whenever the time scale $\mathbb{T}$ is unbounded from above, otherwise, let $\mathbb{T}^{\kappa}=$ $\mathbb{T} \backslash(\rho(\max \mathbb{T}), \max \mathbb{T}]$.

The $\Delta$-derivative of a function $f:[a, b]_{\mathbb{T}} \rightarrow \mathbb{R}$ at a point $t \in \mathbb{T}^{\kappa}$ is defined by

$$
f^{\Delta}(t)=\lim _{s \rightarrow t} \frac{f(\sigma(t))-f(s)}{\sigma(t)-s}, \quad \text { where } s \in \mathbb{T} \backslash\{\sigma(t)\},
$$

provided the above limit exists. If $f^{\Delta}(t)$ exists for each $t \in[a, b]_{\mathbb{T}} \cap \mathbb{T}^{\kappa}$, then $f$ is $\Delta$-differentiable on $[a, b]_{\mathbb{T}}$. In addition, a vector-valued function $f:[a, b]_{\mathbb{T}} \rightarrow \mathbb{R}^{m}$ is $\Delta$-differentiable on $[a, b]_{\mathbb{T}}$, if its coordinate functions $f_{i}:[a, b]_{\mathbb{T}} \rightarrow \mathbb{R}, i=1 \ldots, m$, are $\Delta$-differentiable on $[a, b]_{\mathbb{T}}$. In this case, $f^{\Delta}(t)=\left(f_{1}^{\Delta}(t), \ldots, f_{m}^{\Delta}(t)\right)$. 
It is known that every $\Delta$-differentiable function is continuous (see [4, Theorem 1.16 (i)]) and the linearity of the $\Delta$-derivative holds (see [4, Theorem 1.20]). Moreover, when $\mathbb{T}=\mathbb{R}$, the $\Delta$ differentiability corresponds the usual notion of Frechét differentiability while, for $\mathbb{T}=\mathbb{Z}$, the $\Delta$-derivative is precisely the usual forward difference operator. These facts show how calculus on time scales can unify and extend both continuous and discrete analysis.

Among all available theories of integration on time scales, here we deal with the Riemann $\Delta$-integral. In order to define such notion of integral, let us mention the following result:

For each $\delta>0$, there exists a division $D=\left\{\alpha_{0}, \ldots, \alpha_{\nu(D)}\right\}$ of $[a, b]_{\mathbb{T}}$ such that, for each $j=1, \ldots, \nu(D)$, either $t_{j}-t_{j-1} \leq \delta$ or $t_{j}-t_{j-1}>\delta$ and $\rho\left(t_{j}\right)=t_{j-1}$.

(cf. [5, Lemma 5.7])

For a given $\delta>0, \mathcal{D}_{\delta}=\mathcal{D}_{\delta}[a, b]_{\mathbb{T}}$ denote the set of all divisions of $[a, b]_{\mathbb{T}}$ with the property above.

A bounded function $f:[a, b]_{\mathbb{T}} \rightarrow \mathbb{R}^{m}$ is Riemann $\Delta$-integrable on $[a, b]_{T}$, if there is $I \in \mathbb{R}^{m}$ satisfying: for every $\varepsilon>0$, there exists $\delta>0$ such that

$$
\left|S_{D}(f)-I\right|<\varepsilon
$$

for every Riemann $\Delta$-sum, $S_{D}(f):=\sum_{j=1}^{\nu(D)} f\left(\xi_{j}\right)\left(\alpha_{j}-\alpha_{j-1}\right)$, corresponding to the a division $D \in \mathcal{D}_{\delta}, D=\left\{\alpha_{0}, \ldots, \alpha_{\nu(D)}\right\}$, independent of the way we choose $\xi_{j} \in\left[\alpha_{j-1}, \alpha_{j}\right), j=1, \ldots, \nu(D)$. In this case, $I=\int_{a}^{b} f(t) \Delta t$.

Now, let us consider the linear dynamic equation

$$
y^{\Delta}(t)=P(t) y(t)+h(t), \quad y(a)=\widetilde{x}, \quad t \in[a, b]_{\mathbb{T}},
$$

where $\widetilde{x} \in \mathbb{R}^{n}, P:[a, b]_{\mathbb{T}} \rightarrow L\left(\mathbb{R}^{n}\right)$ and $h:[a, b]_{\mathbb{T}} \rightarrow \mathbb{R}^{n}$ are rd-continuous on $[a, b]_{\mathbb{T}}$. Thanks to the Fundamental Theorem of Calculus (cf. [15, Theorem 4.1]), such initial valued problem can be rewritten as the following integral equation

$$
y(t)=\widetilde{x}+\int_{a}^{t}[P(s) y(s)+h(s)] \Delta s, \quad t \in[a, b]_{\mathbb{T}} .
$$

Besides, a function $y:[a, b]_{\mathbb{T}} \rightarrow \mathbb{R}^{n}$ satisfying the integral equality above is said to be a solution of $(3.1)$ on $[a, b]_{\mathbb{T}}$. 
Quite recently, A. Slavík proved in [39] that the Riemann $\Delta$-integral can be regarded as a special case of the Kurzweil-Stieltjes integral. In addition, in the mentioned paper, a correspondence between dynamic equations on time scales and generalized differential equations is established. Considering the importance of such results to our aim, we summarize them in the following proposition.

Proposition 3.1. Let $\widetilde{\sigma}:(-\infty, \sup \mathbb{T}) \rightarrow \mathbb{T}$ be a function given by

$$
\tilde{\sigma}(t):=\inf \{s \in \mathbb{T}: s \geq t\} .
$$

(i) [39, Theorem 5] Let $f:[a, b]_{\mathbb{T}} \rightarrow \mathbb{R}^{m}$ be an rd-continuous function. Define

$$
F_{1}(t)=\int_{a}^{t} f(s) \Delta s \text { for } t \in[a, b]_{\mathbb{T}}
$$

and

$$
F_{2}(t)=\int_{a}^{t} f(\widetilde{\sigma}(s)) \mathrm{d}[\widetilde{\sigma}(s)] \text { for } t \in[a, b]
$$

Then $F_{2}=F_{1} \circ \tilde{\sigma}$ on $[a, b]$.

(ii) [39, Theorem 12] If $y:[a, b]_{\mathbb{T}} \rightarrow \mathbb{R}^{m}$ is a solution of (3.1), then the function $x=y \circ \widetilde{\sigma}$ : $[a, b] \rightarrow \mathbb{R}^{m}$ is a solution of $(\mathrm{NH})$, where

$$
A(t)=\int_{a}^{t} P(\widetilde{\sigma}(s)) \mathrm{d}[\widetilde{\sigma}(s)] \quad \text { and } \quad f(t)=\int_{a}^{t} h(\widetilde{\sigma}(s)) \mathrm{d}[\widetilde{\sigma}(s)] \quad \text { for } t \in[a, b] .
$$

Symmetrically, if $x:[a, b] \rightarrow \mathbb{R}^{m}$ is a solution of $(\mathrm{NH})$, then the function $y:[a, b]_{\mathbb{T}} \rightarrow \mathbb{R}^{m}$, given by $y(t)=x(t)$ for $t \in[a, b]_{\mathbb{T}}$, is a solution of (3.1).

Remark 3.2. Since the function $\widetilde{\sigma}:[a, b] \rightarrow[a, b]_{\mathbb{T}}$ defined in Proposition (3.1) is clearly monotone and left continuous, it is easy to check that $A:[a, b] \rightarrow L\left(\mathbb{R}^{n}\right)$ and $f:[a, b] \rightarrow \mathbb{R}^{n}$ as given by (3.2) are well-defined, left-continuous and of bounded variation on $[a, b]$.

\subsection{Continuous dependence for dynamical equations on time scales}

In this section, using the results presented in Chapter 2 we derive two theorems on continuous dependence on parameters for dynamic equations on time scales. 
Unlike the usual theory of dynamic equations on time scales (cf. [4]), in the present work, since the solutions of the problems of the type (3.1) are obtained via theory of generalized linear differential equations, no extra assumption over the linear term is needed to ensure the existence of solutions. This means that the $r d$-continuity of the functions in the right-hand side of equations is sufficient for our purposes.

The next theorem is our first outcome in this section and it is based on Theorem 2.7.

Theorem 3.3. Let $P, P_{n}:[a, b]_{\mathbb{T}} \rightarrow L\left(\mathbb{R}^{m}\right)$ and $h, h_{n}:[a, b]_{\mathbb{T}} \rightarrow \mathbb{R}^{m}$, for $n \in \mathbb{N}$, be rd-continuous functions on $[a, b]_{\mathbb{T}}$. Assume $\widetilde{x}, \widetilde{x}_{n} \in \mathbb{R}^{m}, n \in \mathbb{N}$, are such that

$$
\lim _{n \rightarrow \infty}\left|\widetilde{x}_{n}-\widetilde{x}\right|=0
$$

If there is $M>0$ such that

$$
\sup _{t \in[a, b]_{\mathbb{T}}}\left\|P_{n}(t)\right\| \leq M \quad \text { for } n \in \mathbb{N}
$$

and moreover

$$
\begin{array}{r}
\lim _{n \rightarrow \infty} \sup _{t \in[a, b]_{\mathbb{T}}}\left\|\int_{a}^{t}\left(P_{n}(s)-P(s)\right) \Delta s\right\|=0, \\
\lim _{n \rightarrow \infty} \sup _{t \in[a, b]_{\mathbb{T}}}\left|\int_{a}^{t}\left(h_{n}(s)-h(s)\right) \Delta s\right|=0 .
\end{array}
$$

hold, then there exists a solution $y:[a, b]_{\mathbb{T}} \rightarrow \mathbb{R}^{m}$ of (3.1), the initial value problems

$$
y_{n}^{\Delta}(t)=P_{n}(t) y_{n}(t)+h_{n}(t), \quad y_{n}(a)=\widetilde{y}_{n}, \quad t \in[a, b]_{\mathbb{T}}
$$

have solutions $y_{n}:[a, b]_{\mathbb{T}} \rightarrow \mathbb{R}^{m}$ for all $n \in \mathbb{N}$, and

$$
\lim _{n \rightarrow \infty} \sup _{t \in[a, b]_{\mathbb{T}}}\left|y_{n}(t)-y(t)\right|=0 .
$$

Proof. Consider the functions $A \in B V\left([a, b], L\left(\mathbb{R}^{m}\right)\right)$ and $f \in B V\left([a, b], \mathbb{R}^{m}\right)$ given by (3.2) and let $x:[a, b] \rightarrow \mathbb{R}^{m}$ be the solution of $(\mathrm{NH})$, whose existence is guarantee by Proposition 2.1, having in mind Remark 3.2.

For each $n \in \mathbb{N}$ and $t \in[a, b]$, define

$$
A_{n}(t)=\int_{a}^{t} P_{n}(\widetilde{\sigma}(s)) \mathrm{d}[\widetilde{\sigma}(s)] \quad \text { and } \quad f_{n}(t)=\int_{a}^{t} h_{n}(\widetilde{\sigma}(s)) \mathrm{d}[\widetilde{\sigma}(s)] .
$$


Since the functions $A_{n}:[a, b]_{\mathbb{T}} \rightarrow L\left(\mathbb{R}^{m}\right)$ are left-continuous on $(a, b]$ for all $n \in \mathbb{N}$, the generalized linear differential equations (2.25) have solutions $x_{n}:[a, b] \rightarrow \mathbb{R}^{m}, n \in \mathbb{N}$.

Note that, by Proposition 3.1 (i), for each $n \in \mathbb{N}$ and each $t \in[a, b]$,

$$
\begin{aligned}
\left\|A_{n}(t)-A(t)\right\| & =\left\|\int_{a}^{t}\left(P_{n}-P\right)(\widetilde{\sigma}(s)) \mathrm{d}[\widetilde{\sigma}(s)]\right\| \\
& =\left\|\int_{a}^{\widetilde{\sigma}(t)}\left(P_{n}-P\right)(s) \Delta s\right\| \leq \sup _{\tau \in[a, b]_{\mathbb{T}}}\left\|\int_{a}^{\tau}\left(P_{n}-P\right)(s) \Delta s\right\| .
\end{aligned}
$$

Therefore

$$
\left\|A_{n}-A\right\|_{\infty} \leq \sup _{t \in[a, b]_{\mathbb{T}}}\left\|\int_{a}^{t}\left(P_{n}-P\right)(s) \Delta s\right\|, \quad n \in \mathbb{N} .
$$

Analogously,

$$
\left\|f_{n}-f\right\|_{\infty} \leq \sup _{t \in[a, b]_{\mathbb{T}}}\left|\int_{a}^{t}\left(h_{n}-h\right)(s) \Delta s\right|, \quad n \in \mathbb{N} .
$$

Using (3.5), we obtain

$$
\lim _{n \rightarrow \infty}\left\|A_{n}-A\right\|_{\infty}=0 \quad \text { and } \quad \lim _{n \rightarrow \infty}\left\|f_{n}-f\right\|_{\infty}=0
$$

which means that assumptions (2.7) and (2.24) of Theorem 2.7 are satisfied.

On the other hand, given $a \leq c<d \leq b$, by a property of the abstract $K S$-integral (cf. Proposition 1.4 (i)),

$$
\left\|A_{n}(d)-A_{n}(c)\right\|=\left\|\int_{c}^{d} P_{n}(\widetilde{\sigma}(s)) \mathrm{d}[\widetilde{\sigma}(s)]\right\| \leq\left\|P_{n} \circ \widetilde{\sigma}\right\|_{\infty}\left(\operatorname{var}_{c}^{d} \widetilde{\sigma}\right),
$$

holds for each $n \in \mathbb{N}$, where from by (3.4), we get

$$
\operatorname{var}_{a}^{b} A_{n} \leq\left(\sup _{t \in[a, b]_{\mathbb{T}}}\left\|P_{n}(t)\right\|_{L\left(\mathbb{R}^{m}\right)}\right)\left(\operatorname{var}_{a}^{b} \widetilde{\sigma}\right) \leq M\left(\operatorname{var}_{a}^{b} \widetilde{\sigma}\right), \quad \text { for all } n \in \mathbb{N} .
$$

Hence the assumption (2.23) of Theorem 2.7 is satisfied as well. Consequently,

$$
\lim _{n \rightarrow \infty}\left\|x_{n}-x\right\|_{\infty}=0
$$

is true. In view of this, since by Proposition 3.1 (ii), the functions $y, y_{n}:[a, b]_{\mathbb{T}} \rightarrow \mathbb{R}^{m}, n \in \mathbb{N}$, obtained as the restriction of $x$ and $x_{n}$ to $[a, b]_{\mathbb{T}}$ respectively, are the corresponding solutions of (3.1) and (3.6), it follows that (3.7) is also true, which completes the proof. 
Two continuous dependence results for solutions of nonlinear dynamic equations on time scales were presented in [39]. These results derive from Theorems 8.2 and 8.7 from [32] by way of the relation between generalized ODEs and dynamic equations on time scales stated in Theorem 3.3 (ii). In view of Propositions 2.11 and 2.12, Theorem 3.3 provides, for the linear case, a result which generalizes both Theorem 14 and Theorem 16 from [39].

Making use of Corollary 2.5 we obtain the following theorem.

Theorem 3.4. Let $P, P_{n}:[a, b]_{\mathbb{T}} \rightarrow L\left(\mathbb{R}^{m}\right)$ and $h, h_{n}:[a, b]_{\mathbb{T}} \rightarrow \mathbb{R}^{m}$ for $n \in \mathbb{N}$ be rd-continuous functions on $[a, b]_{\mathbb{T}}$ and let $\widetilde{x}, \widetilde{x}_{n} \in \mathbb{R}^{m}, n \in \mathbb{N}$, be given. Assume (3.3) holds and

$$
\begin{aligned}
& \lim _{n \rightarrow \infty}\left[1+\alpha_{n}\right] \sup _{t \in[a, b]_{\mathbb{T}}}\left\|\int_{a}^{t}\left(P_{n}(s)-P(s)\right) \Delta s\right\|=0, \\
& \lim _{n \rightarrow \infty}\left[1+\alpha_{n}\right] \sup _{t \in[a, b]_{\mathbb{T}}}\left|\int_{a}^{t}\left(h_{n}(s)-h(s)\right) \Delta s\right|=0,
\end{aligned}
$$

where $\alpha_{n}=\sup _{t \in[a, b]_{\mathbb{T}}}\left\|P_{n}(t)\right\|_{L\left(\mathbb{R}^{m}\right)}+\sup _{t \in[a, b]]_{\mathbb{T}}}\left\|h_{n}(t)\right\|_{\mathbb{R}^{m}}, n \in \mathbb{N}$. Then equation (3.1) has a solution y, equations (3.6) have solutions $y_{n}$ for all $n \in \mathbb{N}$ and (3.7) holds.

Proof. Consider the functions $A_{n}, A, f_{n}, f, n \in \mathbb{N}$, defined by (3.2) and (3.8). Let $x$ and $x_{n}, n \in \mathbb{N}$, be the solutions of the generalized linear differential equations $(\mathrm{NH})$ and $(2.25)$, respectively. Similarly as in the proof of Theorem 3.3, the estimates (3.9) and (3.10) are true. In addition,

$$
\operatorname{var}_{a}^{b} A_{n} \leq\left(\sup _{t \in[a, b]_{\mathbb{T}}}\left\|P_{n}(t)\right\|_{L\left(\mathbb{R}^{m}\right)}\right)\left(\operatorname{var}_{a}^{b} \widetilde{\sigma}\right) \quad \text { and } \quad \operatorname{var}_{a}^{b} f_{n} \leq\left(\sup _{t \in[a, b]_{\mathbb{T}}}\left\|h_{n}(t)\right\|_{\mathbb{R}^{m}}\right)\left(\operatorname{var}_{a}^{b} \widetilde{\sigma}\right) .
$$

These estimates, together with (3.11), lead to the assumptions of Corollary 2.5. Thus (2.15) holds and we may complete the proof of the theorem using the same argument as in the end of the proof of Theorem 3.3. 



$\frac{100}{4}$

\section{Applications to functional differential equations}

In this chapter, we apply the continuous dependence results obtained in Chapter 2 to Retarded Functional Differential Equations (we write RFDEs for short). The first section of this chapter, presents some preliminary notations and results concerning the relationship between RFDEs and generalized differential equations (e.g. [9], [10], [23] and [29]). In the second section, new results on continuous dependence of solutions on parameters in the framework of RFDEs are obtained.

\subsection{RFDEs and generalized differential equations}

For some fixed $m \in \mathbb{N}$, let $\mathbb{R}^{m}$ be the Euclidean space with norm $|\cdot|$. Recall that $G\left([a, b], \mathbb{R}^{m}\right)$ denotes the Banach space of all regulated functions defined on $[a, b]$ with values in $\mathbb{R}^{m}$, equipped with the induced norm: $\|f\|=\sup _{t \in[a, b]}|f(t)|, f \in G\left([a, b], \mathbb{R}^{m}\right)$. 
In what follows, let us fix $a, b, r \in \mathbb{R}$ with $a<b$ and $0<r<(b-a)$. For $y:[a-r, b] \rightarrow \mathbb{R}^{m}$ and $t \in[a, b]$, define the function $y_{t}:[-r, 0] \rightarrow \mathbb{R}^{m}$, which expresses the history or memory of $y$ on $[t-r, t]$, by

$$
y_{t}(\theta)=y(t+\theta), \quad \theta \in[-r, 0]
$$

It is clear that for $y \in G\left([a-r, b], \mathbb{R}^{m}\right)$ and $t \in[a, b]$, we have $y_{t} \in G\left([-r, 0], \mathbb{R}^{m}\right)$.

Given $f: G\left([-r, 0], \mathbb{R}^{m}\right) \times[a, b] \rightarrow \mathbb{R}^{m}$ and $\phi \in G\left([-r, 0], \mathbb{R}^{m}\right)$, consider the following initial value problem for a retarded functional differential equation

$$
\dot{y}(t)=f\left(y_{t}, t\right), \quad y_{a}=\phi
$$

Recall that a function $y:[a-r, b] \rightarrow \mathbb{R}^{m}$ is a solution of (4.1) on $[a, b]$, if $y \in G\left([a-r, b], \mathbb{R}^{m}\right)$, $y_{a}=\phi$ and the equality $\dot{y}(t)=f\left(y_{t}, t\right)$ holds for almost every $t \in[a, b]$.

Initial value problems for functional differential equations with continuous initial condition were extensively studied by J. Hale in [16]. With regard to linear systems, we can mention the papers [41] and [42] by M. Tvrdý, where the equations have initial data in $B V\left([-r, 0], \mathbb{R}^{m}\right)$. In the present work, we are particularly interested in linear RFDEs

$$
\left\{\begin{array}{l}
\dot{y}(t)=\mathcal{L}\left(y_{t}, t\right) \\
y_{a}=\phi
\end{array}\right.
$$

where $\phi \in G\left([-r, 0], \mathbb{R}^{m}\right)$ and the function $\mathcal{L}: G\left([-r, 0], \mathbb{R}^{m}\right) \times[a, b] \rightarrow \mathbb{R}^{m}$ is linear in the first variable.

An equivalent formulation to problem (4.2) can be written if the mapping $t \longmapsto \mathcal{L}\left(y_{t}, t\right)$ is assumed to be integrable in some sense on $[a, b]$, for every $y \in G\left([a-r, b], \mathbb{R}^{m}\right)$. More precisely,

$$
\left\{\begin{array}{l}
y(t)=\phi(0)+\int_{a}^{t} \mathcal{L}\left(y_{s}, s\right) \mathrm{d} s, \quad t \in[a, b] \\
y_{a}=\phi
\end{array}\right.
$$

Concerning the function $\mathcal{L}: G\left([-r, 0], \mathbb{R}^{m}\right) \times[a, b] \rightarrow \mathbb{R}^{m}$, we assume, for each $y \in G([a-$ $\left.r, b], \mathbb{R}^{m}\right), t \longmapsto \mathcal{L}\left(y_{t}, t\right)$ is Kurzweil-Henstock integrable on $[a, b]$ (see Chapter 1 ) and that the following Lipschitz condition is satisfied: 
(L) There exists $M:[a, b] \rightarrow \mathbb{R}$ Kurzweil-Henstock integrable on $[a, b]$ such that

$$
\left|\int_{t_{1}}^{t_{2}} \mathcal{L}\left(y_{s}-z_{s}, s\right) \mathrm{d} s\right| \leq \int_{t_{1}}^{t_{2}} M(s)\left\|y_{s}-z_{s}\right\| \mathrm{d} s, \quad t_{1}, t_{2} \in[a, b], y, z \in G\left([a-r, b], \mathbb{R}^{m}\right) .
$$

A particular situation in which condition (L) holds is when

$$
\mathcal{L}(\psi, t)=\int_{-r}^{0} d_{\theta}[\eta(t, \theta)] \psi(\theta), \quad t \in[a, b], \psi \in \mathcal{C}\left([-r, 0], \mathbb{R}^{m}\right)
$$

where the function $\eta: \mathbb{R} \times \mathbb{R} \rightarrow L\left(\mathbb{R}^{m}\right)$, which exists by the Riesz Representation Theorem (cf. [40, Theorem III.5.6]), is such that, for each $t$, the function $\eta(t, \cdot)$ is left continuous and of bounded variation on $[-r, 0]$, with $m(t)=\operatorname{var}_{-r}^{0} \eta(t, \cdot)$ being Lebesgue integrable on $[a, b]$. In this case,

$$
|\mathcal{L}(\psi, t)| \leq m(t)\|\psi\|, \quad t \in[a, b], \psi \in \mathcal{C}\left([-r, 0], \mathbb{R}^{m}\right)
$$

As in [9] (see also [10]), the Lipschitz-type condition was imposed to the indefinite integral of $\mathcal{L}$ rather than to the function itself. Such type of condition, together with an assumption of Caratheodory-type, was used by M. Federson and P. Táboas in [9] to prove a correspondence between RFDEs and a certain class of generalized differential equations with values in a Banach space. Notice that, in the linear case, a Caratheodory-type condition holds in the unity ball, by taking $z$ as the null function in (L).

The problem of regarding RFDEs as a class of generalized differential equations was first treated by C. Imaz and Z. Vorel in [23] and by F. Oliva and Z. Vorel in [29]. Based on the ideas coming from the mentioned papers, for $y \in G\left([a-r, b], \mathbb{R}^{m}\right)$ and $t \in[a, b]$, define

$$
F(y, t)(\vartheta)= \begin{cases}0, & a-r \leq \vartheta \leq a, \\ \int_{a}^{\vartheta} \mathcal{L}\left(y_{s}, s\right) \mathrm{d} s, & a \leq \vartheta \leq t \leq b, \\ \int_{a}^{t} \mathcal{L}\left(y_{s}, s\right) \mathrm{d} s, & a \leq t \leq \vartheta \leq b .\end{cases}
$$

It is easy to check that, for each pair $(y, t) \in G\left([a-r, b], \mathbb{R}^{m}\right) \times[a, b]$, the function $F(y, t)$ : $[a-r, b] \rightarrow \mathbb{R}^{m}$ is continuous on $[a-r, b]$, that is, $F(y, t) \in C\left([a-r, b], \mathbb{R}^{m}\right)$.

For each $t \in[a, b]$, consider $A(t): G\left([a-r, b], \mathbb{R}^{m}\right) \rightarrow G\left([a-r, b], \mathbb{R}^{m}\right)$ given by

$$
A(t) y=F(y, t), \text { for } y \in G\left([a-r, b], \mathbb{R}^{m}\right) .
$$


By the Lipschitz condition (L), we have

$$
\|A(t) y\|=\sup _{\vartheta \in[a-r, b]}|F(y, t)(\vartheta)| \leq \sup _{\vartheta \in[a, t]}\left|\int_{a}^{\vartheta} \mathcal{L}\left(y_{s}, s\right) \mathrm{d} s\right| \leq \int_{a}^{t} M(s) \mathrm{d} s\|y\|,
$$

for $y \in G\left([a-r, b], \mathbb{R}^{m}\right)$. Then, in view of the linearity of the function $\mathcal{L}$ and the linearity of the integral, $A(t) \in L\left(G\left([a-r, b], \mathbb{R}^{m}\right)\right)$.

On the other hand, for $a \leq s_{1}<s_{2} \leq b$ and $y \in G\left([a-r, b], \mathbb{R}^{m}\right)$, by (L), we have

$$
\begin{aligned}
\left\|\left[A\left(s_{2}\right)-A\left(s_{1}\right)\right] y\right\| & =\sup _{\vartheta \in[a-r, b]}\left|\left[A\left(s_{2}\right) y\right](\vartheta)-\left[A\left(s_{1}\right) y\right](\vartheta)\right| \\
& =\sup _{\vartheta \in\left[s_{1}, s_{2}\right]}\left|\int_{s_{1}}^{\vartheta} \mathcal{L}\left(y_{s}, s\right) \mathrm{d} s\right| \\
& \leq\left(\int_{s_{1}}^{s_{2}} M(s) \mathrm{d} s\right)\|y\| .
\end{aligned}
$$

Thus, it follows that

$$
\left\|A\left(s_{2}\right)-A\left(s_{1}\right)\right\|_{L\left(G\left([a-r, b], \mathbb{R}^{m}\right)\right)}=\sup _{\|y\| \leq 1}\left\|\left[A\left(s_{2}\right)-A\left(s_{1}\right)\right] y\right\| \leq \int_{s_{1}}^{s_{2}} M(s) \mathrm{d} s
$$

and therefore

$$
\operatorname{var}_{a}^{b} A \leq \int_{a}^{b} M(s) \mathrm{d} s
$$

which implies that the function $A:[a, b] \rightarrow L\left(G\left([a-r, b], \mathbb{R}^{m}\right)\right)$ is of bounded variation. In addition, thanks to the Hake property of the Kurzweil-Henstock integral (see [14, Theorem 9.5]), $\Delta^{-} A(t)=0$, for every $t \in(a, b]$. Hence, condition (E) holds and Proposition 2.1 ensures the existence of a unique solution of the generalized linear differential equation

$$
x(t)=\widetilde{x}+\int_{a}^{t} \mathrm{~d}[A] x, \quad t \in[a, b],
$$

where $\widetilde{x}$ is the function defined on $[a-r, b]$ by

$$
\widetilde{x}(\vartheta)= \begin{cases}\phi(\vartheta-a), & \vartheta \in[a-r, a] \\ \phi(0), & \vartheta \in[a, b]\end{cases}
$$

More generaly, let us consider the perturbed problem

$$
\left\{\begin{array}{l}
\dot{y}(t)=\mathcal{L}\left(y_{t}, t\right)+h(t) \\
y_{a}=\phi
\end{array}\right.
$$


where, as before, $\phi \in G\left([-r, 0], \mathbb{R}^{m}\right), \mathcal{L}: G\left([-r, 0], \mathbb{R}^{m}\right) \times[a, b] \rightarrow \mathbb{R}^{m}$ is linear in the first variable and satisfies (L), and the function $h:[a, b] \rightarrow \mathbb{R}^{m}$ is Kurzweil-Henstock integrable on $[a, b]$. Also, assume the mapping $t \longmapsto \mathcal{L}\left(y_{t}, t\right)$ is Kurzweil-Henstock integrable on $[a, b]$, for every $y \in G\left([a-r, b], \mathbb{R}^{m}\right)$. Hence, we can write the integral form of the perturbed problem as

$$
\left\{\begin{array}{l}
y(t)=\phi(0)+\int_{a}^{t} \mathcal{L}\left(y_{s}, s\right) \mathrm{d} s+\int_{a}^{t} h(s) \mathrm{d} s, \quad t \in[a, b] \\
y_{a}=\phi
\end{array}\right.
$$

Similarly to the homogeneous case, for $t \in[a, b]$, define

$$
f(t)(\vartheta)=\left\{\begin{array}{cl}
0, & a-r \leq \vartheta \leq a \\
\int_{a}^{\vartheta} h(s) \mathrm{d} s, & a \leq \vartheta \leq t \leq b \\
\int_{a}^{t} h(s) \mathrm{d} s, & a \leq t \leq \vartheta \leq b
\end{array}\right.
$$

Note that, by definition, $f(t) \in C\left([a-r, b], \mathbb{R}^{m}\right)$, for every $t \in[a, b]$. Moreover, it is not hard to prove that the function $f:[a, b] \rightarrow G\left([a-r, b], \mathbb{R}^{m}\right)$ is continuous.

Let $A:[a, b] \rightarrow L\left(G\left([a-r, b], \mathbb{R}^{m}\right)\right)$ be given by (4.5). According to Proposition 2.1 and having in mind the properties of the function $A$ described before, there exists a solution $x:[a, b] \rightarrow G\left([a-r, b], \mathbb{R}^{m}\right)$ of the generalized linear differential equation

$$
x(t)=\widetilde{x}+\int_{a}^{t} \mathrm{~d}[A] x+f(t)-f(a), \quad t \in[a, b],
$$

with $\widetilde{x}$ as in (4.9).

The connection between the solutions of (NH) and (4.10) or, in particular, in the homogeneos case, between $(\mathrm{H})$ and (4.3), can be established as in [9] and [10]. In the following lines, we describe this relationship in more details. In order to do that, an auxiliar result, borrowed from [10], is needed (see also [29, Lemma 2.1]). It is important to mention that the proof given in [10] corresponds to a technical manipulation of the solution and does not depend on the conditions of Caratheodory- and Lipschitz-types assumed there. In view of this, we can state [10, Lemma 3.3] for our purposes as follows. 
Lemma 4.1. Assume $x$ is a solution of $(\mathrm{NH})$ on $[a, b]$ with initial condition given by (4.9), where $A$ and $f$ are the functions defined in (4.5) and (4.11) respectively. Then, for all $t \in[a, b]$, we have

$$
x(t)(\tau)=x(\tau)(\tau), \quad \tau \in[a-r, t]
$$

and

$$
x(t)(\tau)=x(t)(t), \quad \tau \in[t, b]
$$

Proposition 4.2. Let $\phi \in G\left([-r, 0], \mathbb{R}^{m}\right)$ and $\mathcal{L}: G\left([-r, 0], \mathbb{R}^{m}\right) \times[a, b] \rightarrow \mathbb{R}^{m}$ be a function linear in the first variable such that $t \longmapsto \mathcal{L}\left(y_{t}, t\right)$ is Kurzweil-Henstock integrable on $[a, b]$ and (L) holds. Assume $x$ is a solution of $(\mathrm{NH})$ on $[a, b]$ with initial condition given by (4.9), where A and $f$ are the functions defined in (4.5) and (4.11) respectively. For $\vartheta \in[a-r, b]$, let

$$
y(\vartheta)= \begin{cases}x(a)(\vartheta), & \vartheta \in[a-r, a], \\ x(\vartheta)(\vartheta), & \vartheta \in[a, b] .\end{cases}
$$

Then $y$ is a solution of (4.10) on $[a-r, b]$.

Proof. First of all, note that, by the definition of $y$ and (4.9), we have $y_{a}=\phi$. Indeed,

$$
y_{a}(\theta)=y(a+\theta)=x(a)(a+\theta)=\widetilde{x}(a+\theta)=\phi(\theta), \quad \text { for all } \theta \in[-r, 0] .
$$

Let us fix an arbitrary $\vartheta \in[a, b]$. By Lemma 4.1, we can write

$$
\begin{aligned}
y(\vartheta)-y(a) & =x(\vartheta)(\vartheta)-x(a)(a) \\
& =x(\vartheta)(\vartheta)-x(a)(\vartheta) \\
& =\left(\int_{a}^{\vartheta} \mathrm{d}[A] x\right)(\vartheta)+[f(\vartheta)-f(a)](\vartheta) . \\
& =\left(\int_{a}^{\vartheta} \mathrm{d}[A] x\right)(\vartheta)+\int_{a}^{\vartheta} h(s) \mathrm{d} s .
\end{aligned}
$$

Given $\varepsilon>0$, since $x:[a, b] \rightarrow G\left([a-r, b], \mathbb{R}^{m}\right)$ is regulated, by [13, Proposition 1.9] (with an obvious extension to Banach space-valued functions), there is a finite sequence, $a=t_{0}<t_{1}<$ $\ldots<t_{n}=\vartheta$, such that

$$
\|x(t)-x(s)\|<\varepsilon, \quad \text { whenever } t_{k-1}<s<t<t_{k} \text { for } k=1, \ldots, n
$$


Consider a gauge $\delta$ on $[a, \vartheta]$ corresponding to the existence of the integral $\int_{a}^{\vartheta} \mathrm{d}[A] x$, that is, a gauge such that

$$
\left\|S(\mathrm{~d} A, x, P)-\int_{a}^{\vartheta} \mathrm{d}[A] x\right\|<\varepsilon, \quad \text { for all } \delta \text {-fine partitions } P \text { of }[a, \vartheta] .
$$

Assume further that

$$
\begin{aligned}
& \delta(\tau)<\min \left\{\frac{t_{k}-t_{k-1}}{2}: k=1, \ldots, n\right\}, \quad \text { for } \tau \in[a, \vartheta] \\
0<\delta(\tau) & <\min \left\{\left|\tau-t_{k}\right|,\left|\tau-t_{k-1}\right|\right\}, \quad \text { for } \tau \in\left(t_{k-1}, t_{k}\right), k=1, \ldots, n .
\end{aligned}
$$

It is easy to check that, by this choice of the gauge, each subinterval of a $\delta$-fine partition contains at most one of the points $t_{0}, \ldots, t_{n}$ and, in this case, it corresponds to the tag of that interval. Moreover, by the continuity of the Kurzweil-Henstock primitive on $[a, \vartheta]$ (cf. [14, Theorem 9.15]), we can assume also that

$$
\left|\int_{t_{k}}^{t_{k}+\delta\left(t_{k}\right)} M(s) \mathrm{d} s\right|<\frac{\varepsilon}{n+2}, \quad \text { for each } k=0,1, \ldots, n .
$$

Having in mind the relations (4.15) and (4.17), for a $\delta$-fine partition $P=\left(\xi_{j},\left[\alpha_{j-1}, \alpha_{j}\right]\right)$ of $[a, \vartheta]$, we get

$$
\begin{aligned}
\left|y(\vartheta)-y(a)-\int_{a}^{\vartheta} \mathcal{L}\left(y_{s}, s\right) \mathrm{d} s-\int_{a}^{\vartheta} h(s) \mathrm{d} s\right| \\
=\left|\left(\int_{a}^{\vartheta} \mathrm{d}[A] x\right)(\vartheta)-\int_{a}^{\vartheta} \mathcal{L}\left(y_{s}, s\right) \mathrm{d} s\right| \\
\leq\left|\left[\int_{a}^{\vartheta} \mathrm{d}[A] x-S(\mathrm{~d} A, x, P)\right](\vartheta)\right|+\left|S(\mathrm{~d} A, x, P)(\vartheta)-\int_{a}^{\vartheta} \mathcal{L}\left(y_{s}, s\right) \mathrm{d} s\right| \\
<\varepsilon+\left|\sum_{j=1}^{\nu(P)}\left[A\left(\alpha_{j}\right)-A\left(\alpha_{j-1}\right)\right] x\left(\xi_{j}\right)(\vartheta)-\int_{a}^{\vartheta} \mathcal{L}\left(y_{s}, s\right) \mathrm{d} s\right| \\
<\varepsilon+\sum_{j=1}^{\nu(P)}\left|\left[A\left(\alpha_{j}\right)-A\left(\alpha_{j-1}\right)\right] x\left(\xi_{j}\right)(\vartheta)-\int_{\alpha_{j-1}}^{\alpha_{j}} \mathcal{L}\left(y_{s}, s\right) \mathrm{d} s\right|
\end{aligned}
$$

For each $j=1, \ldots, \nu(P)$, by the definition of $A$ and taking into account the relations

$$
\begin{aligned}
& x\left(\xi_{j}\right)_{s}=x(s)_{s}=y_{s}, \quad s \in\left[\alpha_{j-1}, \xi_{j}\right], \\
& y_{s}=x(s)_{s}=x\left(\alpha_{j}\right)_{s}, \quad s \in\left[\xi_{j}, \alpha_{j}\right],
\end{aligned} \quad \text { for each } j=1, \ldots, \nu(P),
$$


(cf. Lemma 4.1), it follows that

$$
\begin{aligned}
\mid\left[A\left(\alpha_{j}\right)-\right. & \left.A\left(\alpha_{j-1}\right)\right] x\left(\xi_{j}\right)(\vartheta)-\int_{\alpha_{j-1}}^{\alpha_{j}} \mathcal{L}\left(y_{s}, s\right) \mathrm{d} s \mid \\
= & \left|\int_{\alpha_{j-1}}^{\alpha_{j}} \mathcal{L}\left(x\left(\xi_{j}\right)_{s}, s\right) \mathrm{d} s-\int_{\alpha_{j-1}}^{\alpha_{j}} \mathcal{L}\left(y_{s}, s\right) \mathrm{d} s\right|=\left|\int_{\xi_{j}}^{\alpha_{j}} \mathcal{L}\left(x\left(\xi_{j}\right)_{s}-x\left(\alpha_{j}\right)_{s}, s\right) \mathrm{d} s\right|,
\end{aligned}
$$

where from, using (L), we get

$$
\left|\left[A\left(\alpha_{j}\right)-A\left(\alpha_{j-1}\right)\right] x\left(\xi_{j}\right)(\vartheta)-\int_{\alpha_{j-1}}^{\alpha_{j}} \mathcal{L}\left(y_{s}, s\right) \mathrm{d} s\right| \leq \int_{\xi_{j}}^{\alpha_{j}} M(s)\left\|x\left(\xi_{j}\right)_{s}-x\left(\alpha_{j}\right)_{s}\right\| \mathrm{d} s .
$$

In case $\xi_{j}=t_{k}$, for some $k=0, \ldots, n$, the inequality above, together with (4.18), leads to

$$
\left|\left[A\left(\alpha_{j}\right)-A\left(\alpha_{j-1}\right)\right] x\left(\xi_{j}\right)(\vartheta)-\int_{\alpha_{j-1}}^{\alpha_{j}} \mathcal{L}\left(y_{s}, s\right) \mathrm{d} s\right|<2\|x\| \frac{\varepsilon}{n+2} .
$$

Otherwise, if $\left[\alpha_{j-1}, \alpha_{j}\right]$ does not contain any point of the set $\left\{t_{0}, \ldots, t_{n}\right\}$, we have

$$
\begin{aligned}
\left\|x\left(\xi_{j}\right)_{s}-x\left(\alpha_{j}\right)_{s}\right\| & =\sup _{\theta \in[-r, 0]}\left|x\left(\xi_{j}\right)(s+\theta)-x\left(\alpha_{j}\right)(s+\theta)\right| \\
& =\sup _{\rho \in\left(\xi_{j}, \alpha_{j}\right]}\left|x\left(\xi_{j}\right)(\rho)-x\left(\alpha_{j}\right)(\rho)\right| \\
& \leq \sup _{\rho \in\left(\xi_{j}, \alpha_{j}\right]}\left\{\left|x\left(\xi_{j}\right)(\rho)-x\left(\xi_{j}+\right)(\rho)\right|+\left|x\left(\xi_{j}+\right)(\rho)-x\left(\alpha_{j}\right)(\rho)\right|\right\} \\
& \leq\left\|x\left(\xi_{j}\right)-x\left(\xi_{j}+\right)\right\|+\left\|x\left(\xi_{j}+\right)-x\left(\alpha_{j}\right)\right\|
\end{aligned}
$$

which, by (4.16) and (4.19), implies

$$
\left|\left[A\left(\alpha_{j}\right)-A\left(\alpha_{j-1}\right)\right] x\left(\xi_{j}\right)(\vartheta)-\int_{\alpha_{j-1}}^{\alpha_{j}} \mathcal{L}\left(y_{s}, s\right) \mathrm{d} s\right|<2 \varepsilon \int_{\xi_{j}}^{\alpha_{j}} M(s) \mathrm{d} s .
$$

In summary, thanks to (4.19), (4.20) and (4.21), we have

$$
\begin{aligned}
\left|y(\vartheta)-y(a)-\int_{a}^{\vartheta} \mathcal{L}\left(y_{s}, s\right) \mathrm{d} s-\int_{a}^{\vartheta} h(s) \mathrm{d} s\right| \\
<\varepsilon+\sum_{j=1}^{\nu(P)}\left|\left[A\left(\alpha_{j}\right)-A\left(\alpha_{j-1}\right)\right] x\left(\xi_{j}\right)(\vartheta)-\int_{\alpha_{j-1}}^{\alpha_{j}} \mathcal{L}\left(y_{s}, s\right) \mathrm{d} s\right| \\
<\varepsilon+\sum_{j=1}^{\nu(P)} \int_{\xi_{j}}^{\alpha_{j}} M(s)\left\|x\left(\xi_{j}\right)_{s}-x\left(\alpha_{j}\right)_{s}\right\| \mathrm{d} s \\
<\varepsilon+\sum_{j ;\left[\alpha_{j-1}, \alpha_{j}\right] \cap\left\{t_{0}, \ldots, t_{n}\right\} \neq \emptyset} 2\|x\| \frac{\varepsilon}{2 n+1}+2 \varepsilon \sum_{j ;\left[\alpha_{j-1}, \alpha_{j}\right] \cap\left\{t_{0}, \ldots, t_{n}\right\}=\emptyset} \int_{\xi_{j}}^{\alpha_{j}} M(s) \mathrm{d} s \\
<\varepsilon+2\|x\|(n+1) \frac{\varepsilon}{n+2}+2 \varepsilon \int_{a}^{\vartheta} M(s) \mathrm{d} s
\end{aligned}
$$


that is,

$$
\left|y(\vartheta)-y(a)-\int_{a}^{\vartheta} \mathcal{L}\left(y_{s}, s\right) \mathrm{d} s-\int_{a}^{\vartheta} h(s) \mathrm{d} s\right|<\varepsilon\left(1+2\|x\|+2 \int_{a}^{\vartheta} M(s) \mathrm{d} s\right) .
$$

Then, since $\varepsilon>0$ is arbirtary, the result is proved.

Using these tools, in the next section, we will translate the continuous dependence result obtained in Chapter 2 to the framework of RFDEs.

\subsection{Continuous dependence for linear functional diffe- rential equations}

In this section, we provide new theorems on continuous dependence for linear functional differential equations based on results presented in Chapter 2. More precisely, considering the following initial value problems

$$
\left\{\begin{array}{l}
\dot{y}(t)=\mathcal{L}_{0}\left(\left(y_{0}\right)_{t}, t\right)+h_{0}(t) \\
y_{a}=\phi_{0}
\end{array}, \quad\left\{\begin{array}{l}
\dot{y}_{n}(t)=\mathcal{L}_{n}\left(\left(y_{n}\right)_{t}, t\right)+h_{n}(t) \\
\left(y_{n}\right)_{a}=\phi_{n}
\end{array}, \quad n \in \mathbb{N},\right.\right.
$$

we investigate sufficient conditions ensuring that the sequence of the solutions $y_{n}$ tends to the solution $y$.

In [16], J. Hale presents a well-known result on continuous dependence which corresponds to a natural generalization of the methods from the theory of ordinary differential equations. Roughly speaking, in Theorem 2.2 from [16], it is proved that the solutions depend continuously on a parameter whenever uniqueness is required. However, such result, as the theory developed in [16], is stated for functional differential equations with continuous right-hand sides. Regarding this fact, following results cover rather more general problems.

Concerning the functions $\mathcal{L}_{n}: G\left([-r, 0], \mathbb{R}^{m}\right) \times[a, b] \rightarrow \mathbb{R}^{m}$ and $h_{n}:[a, b] \rightarrow \mathbb{R}^{m}, n \in \mathbb{N} \cup$ $\{0\}$, throughout this section, we assume the following conditions are fulfilled:

1. $\mathcal{L}_{n}$ is linear in the first variable, for all $n \in \mathbb{N} \cup\{0\}$. 
2. For every $y \in G\left([a-r, b], \mathbb{R}^{m}\right)$ and $n \in \mathbb{N} \cup\{0\}$, the mapping $t \longmapsto \mathcal{L}_{n}\left(y_{t}, t\right)$ is KurzweilHenstock integrable on $[a, b]$.

3. For each $n \in \mathbb{N} \cup\{0\}$, there exists $M_{n}:[a, b] \rightarrow \mathbb{R}$ Kurzweil-Henstock integrable on $[a, b]$ such that

$$
\left|\int_{t_{1}}^{t_{2}} \mathcal{L}_{n}\left(y_{s}-z_{s}, s\right) \mathrm{d} s\right| \leq \int_{t_{1}}^{t_{2}} M_{n}(s)\left\|y_{s}-z_{s}\right\| \mathrm{d} s,
$$

for all $t_{1}, t_{2} \in[a, b]$ and all $y, z \in G\left([a-r, b], \mathbb{R}^{m}\right)$.

4. $h_{n}$ is Kurzweil-Henstock integrable on $[a, b]$, for all $n \in \mathbb{N} \cup\{0\}$.

The first result of this section is related to Theorem 2.4 and deals with the homogeneous equation.

Theorem 4.3. Let $\mathcal{L}_{n}: G\left([-r, 0], \mathbb{R}^{m}\right) \times[a, b] \rightarrow \mathbb{R}^{m}, n \in \mathbb{N} \cup\{0\}$, be a sequence of functions which satisfies conditions 1 to 3 . Assume

$$
\lim _{n \rightarrow \infty}\left[1+\int_{a}^{b} M_{n}(s) \mathrm{d} s\right] \sup _{t \in[a, b]}\left|\int_{a}^{t}\left[\mathcal{L}_{n}-\mathcal{L}_{0}\right]\left(y_{s}, s\right) \mathrm{d} s\right|=0
$$

holds for any choice of $y \in G\left([a-r, b], \mathbb{R}^{m}\right)$. Further, consider a sequence $\phi_{n} \in G\left([-r, 0], \mathbb{R}^{m}\right)$, $n \in \mathbb{N} \cup\{0\}$, such that

$$
\lim _{n \rightarrow \infty}\left\|\phi_{n}-\phi_{0}\right\|=0
$$

Then, for each $n \in \mathbb{N} \cup\{0\}$, there exists a solution $y_{n}$ on $[a-r, b]$ of equation

$$
\left\{\begin{array}{l}
y_{n}(t)=\phi_{n}(0)+\int_{a}^{t} \mathcal{L}_{n}\left(\left(y_{n}\right)_{s}, s\right) \mathrm{d} s, \quad t \in[a, b] \\
\left(y_{n}\right)_{a}=\phi_{n},
\end{array}\right.
$$

and $\lim _{n \rightarrow \infty}\left\|y_{n}-y_{0}\right\|_{\infty}=0$.

Proof. To simplify the notation, let $X$ denote the Banach space $G\left([a-r, b], \mathbb{R}^{m}\right)$.

For each $n \in \mathbb{N} \cup\{0\}$, consider the functions $\widetilde{x}_{n} \in X$ and $A_{n}:[a, b] \rightarrow L(X)$ defined as follows

$$
\widetilde{x}_{n}(\vartheta)=\left\{\begin{array}{cl}
\phi_{n}(\vartheta-a), & \vartheta \in[a-r, a] \\
\phi_{n}(0), & \vartheta \in[a, b]
\end{array}\right.
$$

and 


$$
\left[A_{n}(t) y\right](\vartheta)=\left\{\begin{array}{cl}
0, & a-r \leq \vartheta \leq a \\
\int_{a}^{\vartheta} \mathcal{L}_{n}\left(y_{s}, s\right) \mathrm{d} s, & a \leq \vartheta \leq t \leq b \quad, \quad t \in[a, b], \quad y \in X . \\
\int_{a}^{t} \mathcal{L}_{n}\left(y_{s}, s\right) \mathrm{d} s, & a \leq t \leq \vartheta \leq b
\end{array}\right.
$$

Notice that the estimates (4.7) and (4.8) are also true for the functions $A_{n}, n \in \mathbb{N} \cup\{0\}$. Thus, for each $n \in \mathbb{N} \cup\{0\}, A_{n}$ is of bounded variation and left continuous which, by Proposition 2.1, imply the existence of a solution $x_{n}:[a-r, b] \rightarrow X$ of

$$
x_{n}(t)=\widetilde{x}_{n}+\int_{a}^{t} \mathrm{~d}\left[A_{n}\right] x_{n}, \quad t \in[a, b] .
$$

The definition of $A_{n}$, together with condition 3, implies

$$
\begin{aligned}
\left\|A_{n}-A_{0}\right\|_{\infty} & =\sup _{t \in[a, b]}\left\|A_{n}(t)-A_{0}(t)\right\|_{L(X)} \\
& =\sup _{t \in[a, b]}\left(\sup _{\|y\| \leq 1}\left\|A_{n}(t) y-A_{0}(t) y\right\|_{X}\right) \\
& \leq \sup _{\|y\| \leq 1}\left[\sup _{t \in[a, b]}\left|\int_{a}^{t}\left[\mathcal{L}_{n}-\mathcal{L}_{0}\right]\left(y_{s}, s\right) \mathrm{d} s\right|\right] .
\end{aligned}
$$

Moreover, as in (4.8), we also have

$$
\operatorname{var}_{a}^{b} A_{n} \leq \int_{a}^{b} M_{n}(s) \mathrm{d} s, \quad n \in \mathbb{N} .
$$

In view of these inequalities and (4.22), the assumption (2.12) of Theorem 2.4 is satisfied, that is, $\lim _{n \rightarrow \infty}\left\|A_{n}-A_{0}\right\|_{\infty}\left[1+\operatorname{var}_{a}^{b} A_{n}\right]=0$. Besides, (4.23) means that the sequence of initial conditions $\widetilde{x}_{n}$ tends to $\widetilde{x}_{0}$, that is, (2.13) from Theorem 2.4 also holds. Hence,

$$
\lim _{n \rightarrow \infty}\left\|x_{n}-x_{0}\right\|_{\infty}=0
$$

Taking into account Proposition 4.2, for each $n \in \mathbb{N} \cup\{0\}$, problem (4.24) has a solution $y_{n}:[a-r, b] \rightarrow \mathbb{R}^{m}$ given by

$$
y_{n}(\vartheta)= \begin{cases}x_{n}(a)(\vartheta), & \vartheta \in[a-r, a] \\ x_{n}(\vartheta)(\vartheta), & \vartheta \in[a, b]\end{cases}
$$

which, together with (4.28), concludes the proof. 
From Theorem 2.7, we obtain the following continuous dependence result for nonhomogeneous linear RFDEs.

Theorem 4.4. Let $\mathcal{L}_{n}: G\left([-r, 0], \mathbb{R}^{m}\right) \times[a, b] \rightarrow \mathbb{R}^{m}$ and $h_{n}:[a, b] \rightarrow \mathbb{R}^{m}, n \in \mathbb{N} \cup\{0\}$, be functions satisfying conditions 1 to 4 . Consider a sequence $\phi_{n} \in G\left([-r, 0], \mathbb{R}^{m}\right), n \in \mathbb{N} \cup\{0\}$, such that (4.23) holds. Further, assume

$$
\begin{gathered}
\lim _{n \rightarrow \infty}\left\|h_{n}-h_{0}\right\|_{\infty}=0, \\
\lim _{n \rightarrow \infty} \sup _{t \in[a, b]}\left|\int_{t_{0}}^{t}\left[\mathcal{L}_{n}-\mathcal{L}_{0}\right]\left(y_{s}, s\right) \mathrm{d} s\right|=0, \quad \text { for all } y \in G\left([a-r, b], \mathbb{R}^{m}\right), \\
\mu^{*}:=\sup _{n \in \mathbb{N}} \int_{a}^{b} M_{n}(s) \mathrm{d} s<\infty,
\end{gathered}
$$

where, for each $n \in \mathbb{N}, M_{n}$ is the function corresponding to condition 3. Then, for each $n \in$ $\mathbb{N} \cup\{0\}$, there exists a solution $y_{n}$ on $[a-r, b]$ of equation

$$
\left\{\begin{array}{l}
y_{n}(t)=\phi_{n}(0)+\int_{a}^{t} \mathcal{L}_{n}\left(\left(y_{n}\right)_{s}, s\right) \mathrm{d} s+\int_{a}^{t} h_{n}(s) \mathrm{d} s, \quad t \in[a, b], \\
\left(y_{n}\right)_{a}=\phi_{n} .
\end{array}\right.
$$

and $\lim _{n \rightarrow \infty}\left\|y_{n}-y_{0}\right\|_{\infty}=0$.

Proof. To simplify the notation, let $X$ denote the Banach space $G\left([a-r, b], \mathbb{R}^{m}\right)$.

For $n \in \mathbb{N} \cup\{0\}$, let $\widetilde{x}_{n} \in X$ and $A_{n} \in B V([a, b], L(X))$ be given by (4.25) and (4.26) respectively and let $f_{n}:[a, b] \rightarrow X$ be defined by

$$
f_{n}(t)(\vartheta)=\left\{\begin{array}{cl}
0, & a-r \leq \vartheta \leq a, \\
\int_{a}^{\vartheta} h_{n}(s) \mathrm{d} s, & a \leq \vartheta \leq t \leq b, \\
\int_{a}^{t} h_{n}(s) \mathrm{d} s, & a \leq t \leq \vartheta \leq b .
\end{array}\right.
$$

Since $A_{n}$ is left continuous, for each $n \in \mathbb{N} \cup\{0\}$, by Proposition 2.1, there exists a solution $x_{n}:[a, b] \rightarrow X$ of the generalized linear equation

$$
x_{n}(t)=\widetilde{x}_{n}+\int_{a}^{t} \mathrm{~d}\left[A_{n}\right] x_{n}+f_{n}(t)-f_{n}(a), \quad t \in[a, b]
$$

Similarly as in the proof of Theorem 4.3 , we have

$$
\left\|A_{n}-A_{0}\right\|_{\infty} \leq \sup _{\|y\| \leq 1}\left[\sup _{t \in[a, b]}\left|\int_{a}^{t}\left[\mathcal{L}_{n}-\mathcal{L}_{0}\right]\left(y_{s}, s\right) \mathrm{d} s\right|\right] .
$$


which, together with (4.31), implies $\lim _{n \rightarrow \infty}\left\|A_{n}-A_{0}\right\|_{\infty}=0$. Having (4.27) and (4.32) in mind, we get

$$
\sup _{n \in \mathbb{N}} \operatorname{var}_{a}^{b} A_{n} \leq \mu^{*}<\infty .
$$

Moreover, in view of the definition of $f_{n}$, (4.30) yields $\lim _{n \rightarrow \infty}\left\|f_{n}-f_{0}\right\|_{\infty}=0$.

In summary, we have the assumptions of Theorem 2.7 satisfied and (4.28) holds. Therefore, by Proposition 4.2, the solutions $y_{n}$ of (4.33) are given by (4.29), for each $n \in \mathbb{N} \cup\{0\}$. Consequently, $\lim _{n \rightarrow \infty}\left\|y_{n}-y_{0}\right\|_{\infty}=0$.

With respect to continuous dependence on parameters for functional differential equations, let us mention two results inspired in the theory of generalized differential equations. One of them is Theorem 4.1 from [10], which derives from [32, Theorem 8.2] using the correspondence between impulsive RFDEs and generalized differential equations. Secondly, we have Theorem 3.4 from [11] whose proof follows the ideas of the proof of [32, Theorem 8.6], even if it is not obtained via theory of generalized differential equations.

Recalling Proposition 2.11 in Chapter 2, which shows that the assumptions of Theorem 2.7 are more general than those in Theorem 2.9, we can say that, for the linear case, our result (namely, Theorem 4.4) encompasses previous one, as [10, Theorem 4.1] and [11, Theorem 3.4], when no impulses are involved. 

[1] S. M. Afonso; E. Bonotto; M. Federson; Š. Schwabik, Discontinuous local semiflows for Kurzweil equations leading to LaSalle's invariance principle for non-autonomous systems with impulses, J. Differ. Equations 250 (2011), 2969-3001.

[2] R. Agarwal; M. Bohner; D. O'Regan; A. Peterson, Dynamic equations on time scales: a survey, J. Comput. Appl. Math. 141 (2002), 1-26.

[3] M. Ashordia, On the correctness of linear boundary value problems for systems of generalized ordinary differential equations, Proc. Georgian Acad. Sci. Math. 1(4) (1993), $385-394$.

[4] M. Bohner; A. Peterson, Dynamic Equations on Time Scales: An Introduction with Applications. Birkhäuser, Boston, 2001.

[5] M. Bohner; A. Peterson, Advances in Dynamic Equations on Time Scales. Birkhäuser, Boston, 2003.

[6] M. Federson, Fórmulas de substituição para as integrais de gauge, Master Dissertation, Universidade de São Paulo, 1993.

[7] M. Federson, Substitution formulas for the Kurzweil and Henstock vector integrals, Math. Bohem. 127(1) (2002), 15-26. 
[8] M. Federson, Some peculiarities of the Henstock and Kurzweil integrals of Banach space-valued functions, Real Anal. Exchange 29(1) (2003/04), 439-460.

[9] M. Federson; P. Z. Táboas, Topological dynamics of retarded functional differential equations, J. Differ. Equations 195(2) (2003), 313-331.

[10] M. Federson; Š. Schwabik, Generalized ordinary differential equations approach to impulsive retarded functional differential equations, Differential and Integral Equations 19(11) (2006), 1201-1234.

[11] M. Federson; J. G. Mesquita, A new continuous dependence result for impulsive retarded functional differential equations, submitted

[12] D. Fraňková, Continuous dependence on a parameter of solutions of generalized differential equations, Čas. pěst. mat. 114(3) (1989), 230-261.

[13] D. Fraňková, Regulated functions, Math. Bohem. 116(1) (1991), 20-59.

[14] R. A. Gordon, The integrals of Lebesgue, Denjoy, Perron, and Henstock. Graduate Studies in Math., AMS, Providence, Rhode Island, 1994.

[15] G. Sch. Guseinov, Integration on time scales, J. Math. Anal. Appli. 285(1) (2003), 107127.

[16] J. Hale, Theory of Functional Differential Equations. Springer-Verlag, New York, 1977.

[17] Z. Halas, G. A. Monteiro, M. Tvrdý: Emphatic convergence and sequential solutions of generalized linear differential equations, Mem. Differential Equations Math. Phys., 54(2011), 27-49

[18] Z. Halas; M. Tvrdý, Continuous dependence of solutions of generalized linear differential equations on a parameter, Funct. Differ. Equ. 16(2) (2009), 299-313.

[19] R. Henstock, Lectures on the theory of integration. World Scientific, Singapore, 1988.

[20] S. Hilger, Analysis on measures chains - a unified approach to continuous and discrete calculus, Results Math. 18 (1990), 18-56. 
[21] C. S. Hönig, The abstract Riemann-Stieltjes integral and its applications to linear differential equations with generalized boundary conditions. Notas do Instituto de Matemática e Estatística da Universidade de São Paulo, Série Matemática n. 1. 1973.

[22] C. S. Hönig, Volterra Stieltjes-integral equations. Math. Studies 16, North-Holland Publ. Comp., Amsterdam, 1975.

[23] C. Imaz; Z. Vorel, Generalized ordinary differential equations in Banach spaces and applications to functional equations, Bol. Soc. Mat. Mexicana (2) 11 (1966) 47-59.

[24] J. Kurzweil, Generalized ordinary differential equation and continuous dependence on a parameter, Czech. Math. J. 7(82) (1957), 418-449.

[25] J. Kurzweil, Generalized ordinary differential equations, Czech. Math. J. 8(83) (1958), $360-387$.

[26] G. Monteiro; M. Tvrdý, On Kurzweil-Stieltjes integral in Banach space, Math. Bohem., to appear.

[27] G. Monteiro; M. Tvrdý, Generalized linear differential equations in a Banach space: Continuous dependence on a parameter, Discrete and Continuous Dynamical Systems, to appear.

[28] K. M. Naralenkov, On integration by parts for Stieltjes-type integrals of Banach spacevalued functions, Real Anal. Exchange 30 (2004/05), 235-260.

[29] F. Oliva; Z. Vorel, Functional equations and generalized ordinary differential equations, Bol. Soc. Mat. Mexicana (2) 11 (1966), 40-46.

[30] Z. Opial, Continuous parameter dependence in linear systems of differential equations, J. Differ. Equations 3 (1967), 571-579.

[31] S. A. Tikare; M. S. Chaudhary, Henstock-Stieltjes integral for Banach space-valued functions, Bull. Kerala Math. Assoc. 7(2) (2010), 83-92.

[32] Š. Schwabik, Generalized Ordinary Differential Equations. World Scientific, Singapore, 1992. 
[33] ̌̌. Schwabik, Abstract Perron-Stieltjes integral, Math. Bohem. 121(4) (1996), 425-447.

[34] Š. Schwabik, Linear Stieltjes integral equations in Banach spaces, Math. Bohem. 124(4) (1999), 433-457.

[35] Š. Schwabik, Linear Stieltjes integral equations in Banach spaces II: operator valued solutions, Math. Bohem. 125(4) 2000, 431-454.

[36] Š. Schwabik, A note on integration by parts for abstract Perron-Stieltjes integrals, Math. Bohem. 126(3) 2001, 613-629.

[37] Š. Schwabik; G. Ye, Topics in Banach space integration. World Scientific, Singapore, 2005

[38] Š. Schwabik; M. Tvrdý; O. Vejvoda, Differential and Integral Equations: Boundary Value Problems and Adjoint. Academia and Reidel. Praha and Dordrecht, 1979

[39] A. Slavík, Dynamic equations on time scales and generalized ordinary differential equations, J. Math. Anal. Appl. 385 2012, 534-550.

[40] A. Taylor; D. C. Lay, Introduction to Functional Analysis. Wiley, Toronto, 1980.

[41] M. Tvrdý, Linear boundary value type problems for functional differential equations and their adjoints, Czech. Math. J. 25 (1975), 37-66.

[42] M. Tvrdý, Note on functional-differential equations with initial functions of bounded variation, Czech. Math. J. 25(100) (1975), 67-70.

[43] M. Tvrdý, Differential and integral equations in the space of regulated functions, Mem. Differential Equations Math. Phys. 25 (2002), 1-104. 